\title{
Optimal Location of Sources in Transportation Networks
}

\author{
C. H. Yeung and K. Y. Michael Wong \\ Department of Physics, The Hong Kong University of Science and Technology, Hong \\ Kong, China
}

\begin{abstract}
We consider the problem of optimizing the locations of source nodes in transportation networks. A reduction of the fraction of surplus nodes induces a glassy transition. In contrast to most constraint satisfaction problems involving discrete variables, our problem involves continuous variables which lead to cavity fields in the form of functions. The one-step replica symmetry breaking (1RSB) solution involves solving a stable distribution of functionals, which is in general infeasible. In this paper, we obtain small closed sets of functional cavity fields and demonstrate how functional recursions are converted to simple recursions of probabilities, which make the 1RSB solution feasible. The physical results in the replica symmetric (RS) and the 1RSB frameworks are thus derived and the stability of the RS and 1RSB solutions are examined.
\end{abstract}




\section{Introduction}

Constraint satisfaction problems (CSPs), which are highly relevant to many applications such as electronic circuit design and frequency assignment in cellular mobile networks, have been studied in the fields of applied mathematics, computer science and engineering. Despite their usefulness, many CSPs are NP-complete problems [1] associated with algorithmic hardness. It is thus important to understand the physical origin of their hardness and map out the easy and hard regimes for typical instances. Physicists approach the problem by making analogy between CSPs and spin glasses [2, 3]. Objective functions are mapped to spin glass Hamiltonians, enabling the analysis of CSPs using statistical physical techniques. Successful examples are found in the $K$ satisfiability problem [4], graph coloring [5], and vertex cover [6, 7, 8]. They suggest a rich physical picture of CSPs corresponding to the glassy phase in spin glasses.

In this paper we study a problem having a wide range of applications and sharing the characteristics of CSPs. Specifically, we consider the optimal locations of source nodes in transportation networks. Transportation networks consist of nodes with either surplus or deficiency of resources, and an important problem is to distribute them so as to achieve a networkwide satisfaction with a minimum transportation cost [9, 10, 11]. This problem is important in load balancing in computer networks [12] and network flow of commodities [13]. Progress has been made in generalizing the message-passing technique of discrete variables to the passing of cavity energy functions in terms of the continuous current variables [9, 10, 11].

Subsequent work considered networks in which shortages are allowed but cost penalty is imposed [14]. This models applications such as communications networks where shortages are detrimental to the performance of the nodes. Their effects were modeled by step-like shortage costs. This high nonlinearity gives rise to unique behavior and a physical picture absent in the previous models. When the shortage cost is comparable to the transportation cost, the total cost may be optimized either by saving the transportation cost feeding a poor node while sacrificing the satisfaction of the node, or by saving the shortage cost while spending more on the transportation cost. The picture in reminiscent of the learning of noisy examples in perceptrons, where the field distribution of the examples consist of the bands, corresponding to the learned and sacrificed examples respectively [15, 16, 17, 18]. As a result, frustration arises from competition for resources among connected nodes. Numerous metastable states emerge, leading to typical glassy behavior.

The problem of optimal source location in this paper addresses an even more general and practically relevant issue in network design and optimization. Compared with [14] where some nodes remain unsatisfied in the optimized state, this paper moves one step forward and considers the situation in which the location of the source nodes can also be optimized, and all nodes are satisfied. The source location problem has wide applications in the design of optimized transportation networks. For example, the optimal locations of access points in wireless networks can be determined by balancing the signaling cost 
of the access points and the power and bandwidth limitations of the channels linking the mobile subscribers (which can be expressed as the transportation cost).

As demonstrated in [9, 10, 11], the resource allocation problem involves passing messages of continuous variables. When the cost function includes nonlinear terms, the messages generally become extremely complicated. However, as will be described in this paper, there are phases where the space of continuous messages can be replaced by small closed sets of cavity energy functions, and their recursions can be converted to simple recursions of probabilities. In the context of the source location problem, this takes place when the consumer nodes form small clusters surrounded by source nodes. When the ratio of the installation cost of the source nodes and the transportation cost changes, regimes with different maximum cluster sizes are observed, resembling the Devil's staircase observed in the circle map and other dynamical systems [26]. We will show that the use of small closed sets of cavity energy functions is particularly successful in the singlet regime where the consumer nodes are isolated (clusters of size 1) and, when the cost ratio is commensurate, in the doublet regime where the consumer nodes can be paired or isolated.

In fact, clusters formed by similar energetic considerations have been found to play an important role in disordered systems such as the random field Ising model (RFIM) [19], as illustrated in Fig. 1. Indeed, domain sizes in RFIM are determined by the interplay between the random field energy and the domain wall energy, giving rise to the so-called Griffiths singularities and cascades of phase transitions [20, 21]. Analogously, cluster sizes in the source location problem are determined by the balanced between the installation cost of the source nodes and the transportation costs.

Another class of problems that exhibits similar cluster behaviors is the lattice glass models [22, 23, 24]. In the lattice glass models, each site of a network can be occupied or empty, but the number of nearest neighbors cannot exceed a maximum. Analogously, the energetics of cluster formation in the source location problem implies that two neighboring consumer nodes have effective repulsions, since their contiguity prevents them from drawing resources from more neighboring source nodes. However, the present model is richer in behavior, since the cluster energy depends on the current distribution in non-trivial ways, rather than merely counting the number of neighbors. When the particle density in a lattice glass increases, configurations of clusters are formed, causing the system to undergo dynamical freezing transitions preceding the ideal glass transition. Similar transitions will be reported in the source location problem.

The vertex cover problem [6, 8], one of the NP-complete problems in computational complexity theory [1] that attracted recent attention from physicists, also shares similar cluster behaviors. Drawing the analogy of assigning guards in a museum [6], each site of a network can be covered or uncovered, but none of the links can have both vertices uncovered. Hence the uncovered nodes form a configuration with an effective repulsion among them. Indeed, an equivalent configuration of consumer nodes can be found in the singlet regime of the source location problem, since for neighboring consumer nodes, each has one less link to draw resources from the neighboring source nodes. When the 
(a)

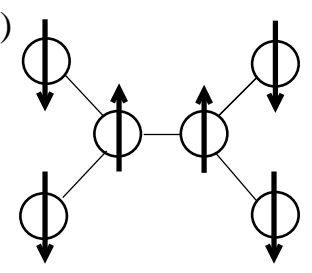

(c)

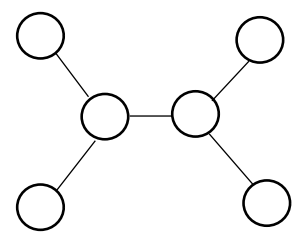

(b)

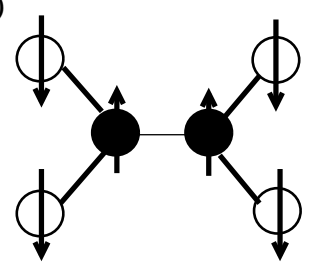

(d)

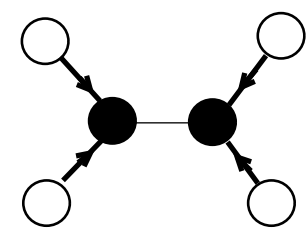

Figure 1. (a)-(b) RFIM of two spins and four fixed boundary spins. Symbols: filled (unfilled) circles -up (down) spins, arrows - direction of random fields of magnitude $h$, thick segments - frustrated couplings of magnitude $J$. When $h / J$ increases from below 2 to above, pattern (a) changes to (b) to form a cluster demarcated by a domain wall of frustrated couplings. (c)-(d) The source location problem defined by Eq. (1) with two nodes and four fixed boundary nodes, and $\Lambda_{i}=-1$ for all $i$. Symbols: filled (unfilled) circles - consumer (source) nodes, arrows - current flows. When $u^{-1}$ decreases from above $\sqrt{2}$ to below, pattern (c) changes to (d) to form a cluster demarcated by a "domain wall" of inward current flow.

average connectivity of the network increases, we will show that it undergoes a glassy transition analogous to that in the vertex cover problem.

The origin of these interesting phenomena can be traced to the presence of frustrations, which refer to the conflicts between competing interaction energies in the system [25]. This connects our problem with a broad class of network CSPs in which frustrations are inherent. When the system consists of numerous states, the replica symmetry-breaking (RSB) solution is applicable, but the distribution of the cavity energy functions is in general infeasible to solve. Nevertheless, with the small closed set of cavity energy functions introduced in this paper, the one-step RSB (1RSB) solution becomes computationally feasible. The physical results based on the replica symmetric (RS) ansatz and the 1RSB configurational entropy are thus derived.

The paper is organized as follows. We introduce our problem in Section 2, presenting simulation results of the optimal behaviors. In Section [3, we discuss the general RS formalism and derive the piecewise quadratic ansatz of the cavity energy functions. In Section 4, we obtain a small closed set of cavity energy functions in the singlet regime and demonstrate how functional recursions are converted to recursions of probabilities. The average energy, the fraction of soft nodes and the RS-RSB phase diagram are derived. In Section 6, we apply the small closed set of cavity fields to the 1RSB formalism and obtain results of the configurational entropy. The conclusion

is given in Section 7. In Appendix A, we describe how the small closed set of cavity energy functions can be applied to the doublet regime in the commensurate case, and the corresponding RS and 1RSB results are derived. 


\begin{tabular}{|c|c|c|c|}
\hline$\Lambda_{i}$ & before optimization & $\xi_{i}$ & after optimization \\
\hline$\geq 0$ & surplus node & $\geq 0$ & $\begin{array}{c}\text { source node } \\
\text { (resource } \\
\text { providing) }\end{array}$ \\
\hline$<0$ & deficient node & $<0^{\dagger}$ & $\geq 0$ \\
\cline { 3 - 4 } & & $\begin{array}{c}\text { consumer node } \\
\text { (consuming) }\end{array}$ \\
\hline
\end{tabular}

${ }^{\dagger}$ The deficient nodes with $\xi_{i}<0$ after optimization are converted to source nodes.

Table 1. A summary of glossary used in this paper.

\section{The Model}

\subsection{Model Formulation}

We consider a network of $N$ nodes, labelled $i=1 \ldots N$. Each node $i$ is connected randomly to a set $\mathcal{L}_{i}$ of $K$ neighbors. Each node $i$ has capacity $\Lambda_{i}$; nodes with positive and negative values of $\Lambda_{i}$ correspond to surplus and deficient nodes respectively. The capacities $\Lambda_{i}$ are randomly drawn from a distribution of $\rho\left(\Lambda_{i}\right)$. With network applications in mind, we consider a bimodal distribution in which $\Lambda_{i}=A(\gg 1)$ with probability $\phi_{s} \geq 0$ and $\Lambda_{i}=-1$ with probability $\phi_{d} \equiv 1-\phi_{s}$. Naturally, the surplus nodes serve as source nodes providing resources to the consumer nodes. However, to minimize cost functions that include transportation costs, it is often desirable to convert some deficient nodes into source nodes as well. Hence in general, the task is to optimally locate these extra source nodes so as to minimize the total cost function. The relevant glossary used in this paper is summarized in Table 1.

We first consider the minimization of the cost function in [14], whose optimization variables are the currents $y_{i j} \equiv-y_{j i}$ of real values from node $j$ to node $i$,

$$
E=\frac{u^{2}}{2} \sum_{i} \Theta\left(-\xi_{i}\right)+\sum_{(i j)} \frac{y_{i j}^{2}}{2} .
$$

$\xi_{i} \equiv \Lambda_{i}+\sum_{j \in \mathcal{L}_{i}} y_{i j}$ is the final resource of node $i$, and $\Theta(x)=1$ when $x>0$, and 0 otherwise. The link connecting nodes $i$ and $j$ is denoted as $(i j)$. The first term corresponds to the unsatisfaction cost imposed on nodes with negative final resource. The second term is the transportation cost. This cost function models load balancing situations in which insufficient provision of resources to a deficient node produces detrimental effects on it (irrespective of the magnitude of insufficiency).

The key to applying the cost function in Eq. (1) to optimize the location of source nodes is to note that once the final resource of a deficient node is negative, 
the unsatisfaction cost remains the same even when its resources are maximally drawn by other nodes of the network. Hence the deficient node effectively becomes a resource provider. Ref. [14] contains many such examples. If we consider the coefficient $u^{2} / 2$ to be the installation cost of a source node, then we can solve the optimal source location problem by first minimizing the cost function in Eq. (11), then identifying the deficient nodes whose final resources are negative, and converting them to source nodes.

Formally, in the optimal source location problem, we introduce the state variables $s_{i}= \pm 1$ for deficient nodes when node $i$ is a consumer or a source node respectively. The cost function is then

$$
E=\frac{u^{2}}{4} \sum_{i \in \mathcal{N}_{D}}\left(1-s_{i}\right)+\sum_{(i j)} \frac{y_{i j}^{2}}{2},
$$

subject to $\xi_{i} \geq 0$ for $s_{i}=1$. No constraints are imposed on nodes with $s_{i}=-1$, since an arbitrary amount of resource can be provided when they are converted to source nodes. $\mathcal{N}_{D}$ is the set of deficient nodes.

To check the equivalence between the cost functions in Eqs. (11) and (2), we can easily see that when $s_{i}=1$, the installation (or unsatisfaction) cost vanishes in both cost functions. When $s_{i}=-1$, we only have to consider the case $\xi_{i}<0$, and the installation (or unsatisfaction) cost is $u^{2} / 2$ in both cost functions. This is because when $\xi_{i} \geq 0$, we can set $s_{i}=1$ to minimize the total cost.

Note that the cost function of the optimal source location problem is identical to that in [14, but the interpretation is far more relevant to network applications. All previous results on networks with nodes of negative capacity can be directly mapped to networks whose unsatisfied nodes are replaced by source nodes. For example, the singlesat regime studied in [14] corresponds to the case that each consumer node is surrounded by source nodes, since the installation cost is low compared with the transportation cost. When the installation cost is gradually raised, resource provision is achieved with less source nodes, but optimization requires the consumer nodes to be located in clusters surrounded by source nodes, forming the clusters observed in [14].

To formulate an algorithm, we introduce the constraints $s_{i} \xi_{i} \geq 0$ for each deficient node. These contraints are not applied to surplus nodes as they are always satisfied. Introducing Lagrange multipliers $\mu_{i}$ for the resource constraint, we minimize the Lagrangian

$$
L=\frac{u^{2}}{4} \sum_{i \in \mathcal{N}_{D}}\left(1-s_{i}\right)+\sum_{i \in \mathcal{N}_{D}} \mu_{i} s_{i} \xi_{i}+\sum_{(i j)} \frac{y_{i j}^{2}}{2}
$$

with the Kühn-Tucker conditions $\mu_{i} s_{i} \xi_{i}=0$ and $\mu_{i} \leq 0$. Optimizing $L$ with respect to $y_{i j}$, one obtains $y_{i j}=\mu_{j} s_{j}-\mu_{i} s_{i}$ and $\mu_{i}=\min \left[0,\left(\Lambda_{i}+\sum_{j \in \mathcal{L}_{i}} \mu_{j} s_{j}\right) / s_{i} c\right]$. Given a particular set of $\left\{s_{i}\right\}$, we iterate these equations to find the corresponding set of $\left\{\mu_{i}\right\}$. The set of optimal $\left\{s_{i}\right\}$ is found by an approach similar to the the GSAT algorithm [31], by comparing the Lagrangian in Eq. (3) for each choice of $\left\{s_{i}\right\}$. In each step of this algorithm, a cluster of $N_{\text {flip }}$ nodes is randomly selected. The network energies involving 


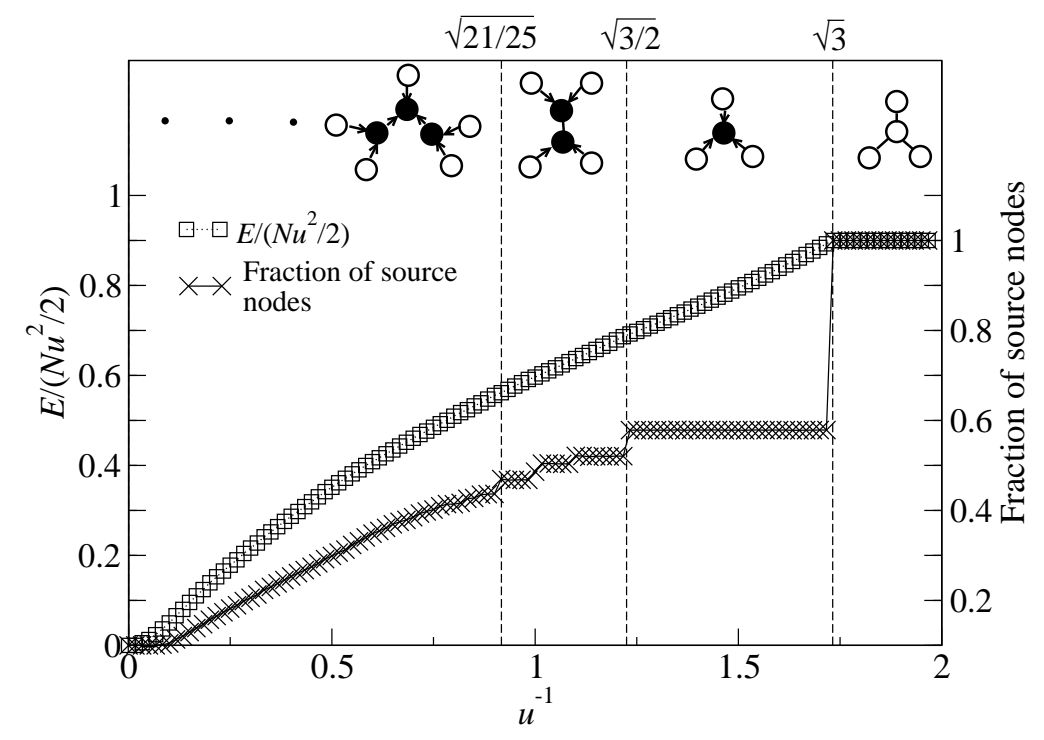

Figure 2. Simulation results of average energy per node and the fraction of network nodes acting as source nodes. Parameters: $K=3, \phi_{d}=0.9, N=100, N_{\text {flip }}=4,100$ samples and 1000 flips. New clusters formed on increasing $u^{-1}$ are sketched at the top, with filled and unfilled circles representing consumer and source nodes respectively.

the different configurations of this cluster are compared, and the cluster configuration is updated to the one that yields the lowest network energy [14].

\subsection{Major Simulation Results}

As shown in Fig. 2 for $K=3$, two phases can be identified: (1) all-source phase for $u^{-1} \geq \sqrt{3}$, in which all nodes are assigned to be source nodes due to the very high transportation cost; (2) partial-source phase for $0<u^{-1}<\sqrt{3}$, in which only some nodes are assigned to be source nodes. (In [14] we also identified a phase transition at $u^{-1}=0$ to an all-consumer phase.)

The fraction of source nodes is a discontinuous function of $u^{-1}$, showing abrupt jumps at threshold values of $u^{-1}$. The step size of the curve decreases as $u^{-1}$ increases, and gradually becomes unresolvable by the numerical experiments. This resembles the Devil's staircase observed in the circle map and other dynamical systems [26]. These threshold values of $u^{-1}$ mark the positions at which certain configurations of the source and consumer nodes become energetically stable. Similar features are observed in RFIM due to the formation of ferromagnetic clusters resultant from the competition between the strengths of couplings and random fields [20, 21]. Except for a shift of the average energy per node, these features are qualitatively similar to the simulation results of [14] which correspond to the case $\phi_{d}=1$, if the latter is reinterpreted from the perspective of the source location problem.

Measuring the average maximum cluster size of the consumer nodes in the samples, we observe abrupt jumps of the cluster size at the same threshold values. This indicates that new types of clusters are formed at each jump, as sketched in the top of Fig. 2 . 


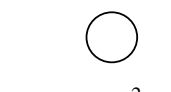

(a) $\varepsilon_{0}=u^{2} / 2$

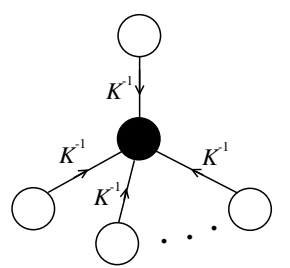

(b) $\mathcal{E}_{1}=(2 K)^{-1}$

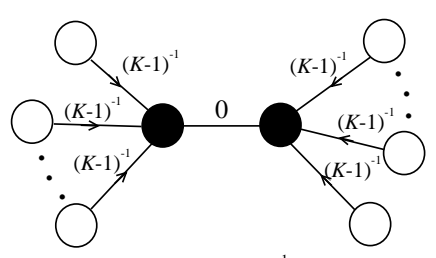

(c) $\mathcal{E}_{2}=(K-1)^{-1}$

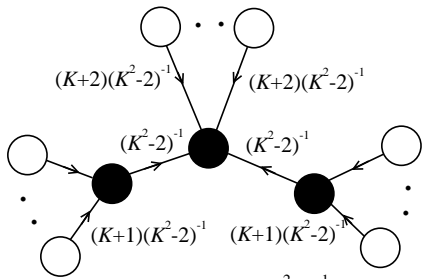

(d) $\mathcal{E}_{3}=(3 K / 2+2)\left(K^{2}-2\right)^{-1}$

Figure 3. Cluster of (a) source node, (b) singly consuming node, (c) doubly consuming nodes, and (d) triply consuming nodes.

The observed threshold values can be calculated by considering the energies of consumer clusters surrounded by source nodes as shown in Fig. 3, obtained by the minimization of Eq. (1). By comparing the energy of different configurations, we have

$$
\begin{array}{ll}
\mathcal{E}_{0} \geq \mathcal{E}_{1} & \text { (singlet) }, \\
\mathcal{E}_{0}+\mathcal{E}_{1} \geq \mathcal{E}_{2} & \text { (doublet) }, \\
\mathcal{E}_{0}+2 \mathcal{E}_{1} \geq \mathcal{E}_{3} & \text { (triplet) },
\end{array}
$$

resulting in the threshold values in Fig. 2. These results agree with those obtained through the cavity approach in Section 3. We call the regime $\sqrt{3 / 2}<u^{-1}<\sqrt{3}$ with isolated consumer nodes the singlet regime, and $\sqrt{21 / 25}<u^{-1}<\sqrt{3 / 2}$ the doublet regime. The isolated nodes are referred to as singly consuming, while the paired consumer nodes doubly consuming.

\section{The Replica Symmetric Ansatz}

\subsection{The RS Recursion at Zero-temperature Limit}

We apply the cavity method [2, 3] assuming that the network has a locally tree-like structure. We denote as $E_{j}\left(y_{j}\right)$ the energy of the tree terminated at node $j$ in the absence of its ancestor node $i$, when a current $y_{j}$ is drawn from $j$ to its ancestor. Relabeling the descendents of $j$ as $k=1, \ldots, K-1, E_{j}\left(y_{j}\right)$ is expressed as

$$
E_{j}\left(y_{j}\right)=\mathcal{H}\left(E_{k=1}, \ldots, E_{K-1} ; \Lambda_{j}, y_{j}\right) .
$$

The functional $\mathcal{H}$ is given by

$$
\begin{aligned}
& \mathcal{H}\left(E_{1}, \ldots, E_{K-1} ; \Lambda_{j}, y_{j}\right) \\
& \equiv \min _{\left\{y_{k}\right\}}\left[\sum_{k \in \mathcal{\mathcal { L } _ { j } \backslash \{ i \}}} E_{k}\left(y_{k}\right)+\frac{u^{2}}{2} \Theta\left(-\Lambda_{j}-\sum_{k \in \mathcal{L}_{j} \backslash\{i\}} y_{k}+y_{j}\right)+\frac{y_{j}^{2}}{2}\right] .
\end{aligned}
$$

In the absence of node $i$, there is no supply or demand of resources through the cavity and the last term $y_{j}^{2} / 2$ should be absent. However, the presence of the extra term results in a clear interpretation of $E_{j}$, as we will see in the following sections. Care has to be taken when dealing with the change of the cavity energy, where $y_{j}$ is taken to be zero to eliminate the effect of the extra transportation cost on the dangling bond. 
We note that $E_{j}$ is an extensive quantity that depends on size of the tree. To formulate a recursion of an intensive energy, we write $E_{j}\left(y_{j}\right)$ as a sum of two terms,

$$
E_{j}\left(y_{j}\right)=E_{j}^{V}\left(y_{j}\right)+E_{j}(0) .
$$

We call $E_{j}^{V}\left(y_{j}\right)$ the cavity energy functions which correspond to the cavity fields in the language of the cavity approach, and represent the energy variation from $E_{j}(0)$, as $y_{j}$ varies. In this case, $E_{j}^{V}(0)=0 . E_{j}(0)$ corresponds to the energy of the tree when no current is drawn from the vertex. We further define the energy change $\Delta E_{j}$ due to the addition of a vertex,

$$
\Delta E_{j}=E_{j}(0)-\sum_{k \in \mathcal{L}_{j} \backslash\{i\}} E_{k}(0)
$$

which simplifies Eq. (5) to

$$
E_{j}^{V}\left(y_{j}\right)=\mathcal{H}\left(E_{k=1}^{V}, \ldots, E_{K-1}^{V} ; \Lambda_{j}, y_{j}\right)-\Delta E_{j}
$$

where

$$
\Delta E_{j}=\mathcal{H}\left(E_{1}^{V}, \ldots, E_{K-1}^{V} ; \Lambda_{j}, 0\right) .
$$

We have thus separated the energy contribution due to the addition of a new vertex from the energy variation due to the changes in the current drawn from the tree.

The distribution $\mathcal{P}\left[E^{V}\right]$ of $E^{V}$ over the vertices of the tree is given by the solution of

$$
\begin{aligned}
\mathcal{P}\left[E_{j}^{V}\right]= & \int d \Lambda_{j} \prod_{k=1}^{K-1} \int d E_{k}^{V} \mathcal{P}\left[E_{k}^{V}\right] \delta\left[E_{j}^{V}-\mathcal{H}\left(E_{1}^{V}, \ldots, E_{K-1}^{V} ; \Lambda_{j}, y_{j}\right)\right. \\
& \left.+\Delta E\left(E_{1}^{V}, \ldots, E_{K-1}^{V} ; \Lambda_{j}\right)\right] .
\end{aligned}
$$

To elucidate the physical behavior of the system, we consider a node fed by $K$ trees forming a Bethe lattice. For instance, we consider the average energy per node. The change in energy due to the additional node $i$ is given by

$$
\Delta \mathcal{E}_{\text {node }}=\mathcal{H}\left(E_{1}^{V}, \ldots, E_{K}^{V} ; \Lambda_{j}, 0\right) .
$$

Similarly, we can consider a link bridging two trees forming a Bethe lattice. The energy change due to the addition of a link between nodes $L$ and $R$ is given by

$$
\Delta \mathcal{E}_{\text {link }}\left(E_{L}^{V}, E_{R}^{V}\right)=\min _{y}\left[E_{L}^{V}(y)+E_{R}^{V}(-y)-\frac{y^{2}}{2}\right] .
$$

Denoting $\langle\ldots\rangle$ as the average over the capacities, the average energy per node is given by

$$
\langle\Delta \mathcal{E}\rangle=\left\langle\Delta \mathcal{E}_{\text {node }}\right\rangle-\frac{K}{2}\left\langle\Delta \mathcal{E}_{\text {link }}\right\rangle
$$

\subsection{The Piecewise Quadratic Solution}

Due to the quadratic form of the transportation cost assumed in Eq. (11), we propose that the cavity energy functions are continuous and piecewise quadratic, namely,

$$
E_{k}^{V}\left(y_{k}\right)=\min _{n_{k}}\left[f_{n_{k}}^{k}\left(y_{k}\right)\right]
$$




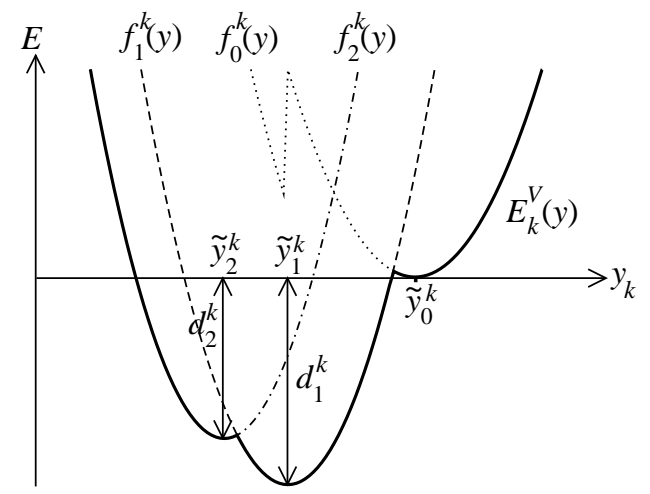

Figure 4. An example of $E_{k}^{V}(y)$ composed of three quadratic functions $f_{n_{k}}^{k}(y)$ labelled by $n_{k}=0,1,2$. Each composite function is characterized by its minimum position $\tilde{y}_{n_{k}}^{k}$, minimum value $d_{n_{k}}^{k}$, and curvature $a_{n_{k}}^{k}$.

where $n_{k}=0,1,2, \ldots$ Indeed, the recursive nature of the quadratic cavity energy functions have been fully employed in deriving the message-passing approach in [9, 10]. We note in passing that a similar recursive structure was used in the Gaussian Belief Propagation algorithm [27] and applied to processing continuous signals such as those in CDMA multiuser detection [28. As a step forward, the ansatz in Eq. (15) further captures the multi-valley features in $E_{k}^{V}\left(y_{k}\right)$, which is crucial in formulating the cavity messages for the present model.

We call $f_{n_{k}}^{k}$ the $n_{k^{-}}$th composite function of $E_{k}\left(y_{k}\right)$. For $n_{k}>0, f_{n_{k}}^{k}\left(y_{k}\right)$ is a quadratic function of the form

$$
f_{n_{k}}^{k}\left(y_{k}\right)=a_{n_{k}}^{k}\left(y_{k}-\tilde{y}_{n_{k}}^{k}\right)^{2}+d_{n_{k}}^{k} .
$$

whereas for $n_{k}=0$, to takes the form

$$
f_{0}^{k}\left(y_{k}\right)=\frac{y_{k}^{2}}{2}+c_{k}+\frac{u^{2}}{2} \Theta\left(y_{k}-\alpha_{k}\right) .
$$

The form of $f_{0}$ is relevant when node $k$ is a source node. Though $f_{0}$ is discontinuous, we will show that the resulting $E^{V}\left(y_{k}\right)$ is continuous since the discontinuity at $y_{k}=\alpha_{k}$ is masked by other quadratic functions. An example of a cavity energy function $E_{k}^{V}\left(y_{k}\right)$ composed of three composite functions is shown in Fig. 4.

We denote the $f_{n_{k}}^{k}$ with the minimum $d_{n_{k}}^{k}$ among all composite functions in $E_{k}^{V}$ as $f_{n_{k}^{*}}^{k}$, i.e.,

$$
n_{k}^{*}=\arg \left(\min _{n_{k}} d_{n_{k}}^{k}\right)
$$

$n_{k}^{*}$ is particularly relevant when we evaluate the energy of the system in the case that the ancestor node of $k$ is a source node. In this case, the resource of the ancestor can be freely drawn by node $k$ without any consequences to other parts of the network, and the optimal current $y_{k}$ takes the value $\tilde{y}_{n_{k}^{*}}^{k}$.

As will be shown in the next subsection, only a few composite functions are relevant in the singlet and the doublet regimes. Each composite function has its fixed values of $\tilde{y}_{n_{k}}^{k}$ and $a_{n_{k}}^{k}$ independent of $k$, but the constant terms is $k$-dependent. Thus, the functional 


\begin{tabular}{|c||c|c|}
\hline$n$ & $\tilde{y}_{n}$ & $a_{n}$ \\
\hline \hline 0 & 0 & $\frac{1}{2}$ \\
\hline 1 & $-K^{-1}$ & $\frac{K}{2(K-1)}$ \\
\hline 2 & $-(K-1)^{-1}$ & $\frac{K^{2}-1}{2\left(K^{2}-K-1\right)}$ \\
\hline
\end{tabular}

Table 2. The table of cavity composite functions $f_{n}(y) \doteq\left(\tilde{y}_{n}, a_{n}\right)$ from $n=0$ to 2 .

form of $E_{k}^{V}\left(y_{k}\right)$ is effectively parametrized by the constant terms $d_{n_{k}}^{k}$ of the composite functions, as given by

$$
E_{k}^{V}\left(y_{k}\right) \doteq\left(d_{0}^{k}, d_{1}^{k}, d_{2}^{k}, \ldots\right)
$$

The relevant composite functions in the singlet and doublet regimes are shown in Table 2 .

\subsection{The Recursion of $E^{V}$}

Using the piecewise quadratic ansatz in Eq. (15), the recursion of $E^{V}$ in Eq. (9) becomes

$$
E_{j}^{V}\left(y_{j}\right)=\min _{\left\{n_{k}\right\}} \mathcal{H}\left(f_{n_{1}}^{k=1}, \ldots, f_{n_{K-1}}^{K-1} ; \Lambda_{j}, y_{j}\right)-\min _{\left\{n_{k}\right\}} \Delta E_{j}\left(f_{n_{1}}^{k=1}, \ldots, f_{n_{K-1}}^{K-1} ; \Lambda_{j}\right) .
$$

We first consider the recursions for a deficient node $j$. Suppose the node is assigned to be a source node, i.e., $\Lambda_{j}+\sum_{k \in \mathcal{L}_{j} \backslash\{i\}} y_{k}-y_{j}<0$. In this case,

$$
\begin{aligned}
& \left.\mathcal{H}\left(f_{n_{1}}^{1}, \ldots, f_{n_{K-1}}^{K-1} ; \Lambda_{j}, y_{j}\right)\right|_{\Lambda_{j}+\sum_{k \in \mathcal{L}_{j} \backslash\{i\}} y_{k}-y_{j}<0} \\
& =\frac{y_{j}^{2}}{2}+\frac{u^{2}}{2} \Theta\left(y_{j}-\Lambda_{j}-\sum_{k \in \mathcal{L}_{j} \backslash\{i\}} \tilde{y}_{n_{k}}^{k}\right)+\sum_{k \in \mathcal{L}_{j} \backslash\{i\}} d_{n_{k}}^{k},
\end{aligned}
$$

which is exactly the form of $f_{0}$ in Eq. (17). The combination $\left\{n_{k}^{*}\right\}$ of composite function minimizes the last term in Eq. (21). Subject to a vertical shift by $\Delta E_{j}$, Eq. (21) is taken to be $f_{0}^{j}$ in Eq. (17) characterized by

$$
d_{0}^{j}=\frac{u^{2}}{2}+\sum_{k \in \mathcal{L}_{j} \backslash\{i\}} d_{n_{k}^{*}}^{k}-\Delta E_{j}
$$

and

$$
\alpha_{j}=\Lambda_{j}+\sum_{k \in \mathcal{L}_{j} \backslash\{i\}} \tilde{y}_{n_{k}^{*}}^{k}
$$

For the moment, we ignore the possibility that combinations other than $\left\{n_{k}^{*}\right\}$ may result in further discontinuity in $f_{0}$, as we will show that composite functions $f_{n}$ with $n>0$ eliminate the effect of the discontinuities. 
Next we suppose the node remains as a consumer node. In this case, $\Lambda_{j}+$ $\sum_{k \in \mathcal{L}_{j} \backslash\{i\}} y_{k}-y_{j}=0$, and $\min _{\left\{n_{k}\right\}} \mathcal{H}\left(f_{n_{1}}^{k=1}, \ldots, f_{n_{K-1}}^{K-1} ; \Lambda_{j}, y_{j}\right)$ is computed subject to this equality constraint,

$$
\begin{aligned}
& \left.\mathcal{H}\left(f_{n_{1}}^{k=1}, \ldots, f_{n_{K-1}}^{K-1} ; \Lambda_{j}, y_{j}\right)\right|_{\Lambda_{j}+\sum_{k \in \mathcal{L}_{j} \backslash\{i\}}} y_{k}-y_{j}=0 \\
& =A_{\left\{n_{k}\right\}}\left(y_{j}-\tilde{Y}_{\left\{n_{k}\right\}}\right)^{2}+D_{\left\{n_{k}\right\}}+\sum_{k \in \mathcal{L}_{j} \backslash\{i\}} d_{n_{k}}^{k} .
\end{aligned}
$$

where

$$
\begin{aligned}
A_{\left\{n_{k}\right\}} & =\frac{1}{2}\left[1+\frac{1}{\sum_{k \in \mathcal{L}_{j} \backslash\{i\}}\left(2 a_{n_{k}}^{k}\right)^{-1}}\right], \\
\tilde{Y}_{\left\{n_{k}\right\}} & =\frac{\Lambda_{j}+\sum_{k \in \mathcal{L}_{j} \backslash\{i\}} \tilde{y}_{n_{k}}^{k}}{1+\sum_{k \in \mathcal{L}_{j} \backslash\{i\}}\left(2 a_{n_{k}}^{k}\right)^{-1}}, \\
D_{\left\{n_{k}\right\}} & =\frac{\left(\Lambda_{j}+\sum_{k \in \mathcal{L}_{j} \backslash\{i\}} \tilde{y}_{n_{k}}^{k}\right)^{2}}{2\left[1+\sum_{k \in \mathcal{L}_{j} \backslash\{i\}}\left(2 a_{n_{k}}^{k}\right)^{-1}\right]},
\end{aligned}
$$

and the optimal currents drawn from the descendents are

$$
y_{k,\left\{n_{k}\right\}}^{*}=\frac{y_{j}-\Lambda_{j}-\sum_{l \in \mathcal{L}_{j} \backslash\{i\}} \tilde{y}_{n_{l}}^{l}}{2 a_{k} \sum_{l \in \mathcal{L}_{j} \backslash\{i\}}\left(2 a_{n_{l}}^{l}\right)^{-1}}+\tilde{y}_{k} .
$$

Next, we consider the recursions for a source node $j$. In this case, the cavity energy function consists of the composite function with $n_{j}=0$ only, with

$$
d_{0}^{j}=\sum_{k \in \mathcal{L}_{j} \backslash\{i\}} d_{n_{k}^{*}}^{k}-\Delta E_{j}
$$

Table 3 summarizes the combinations $\left\{n_{k}\right\}$ which lead to the composite functions $n_{j}=1,2$ in Table 2, enabling us to analyze the singlet and doublet regimes. We represent these relations of $\left\{n_{k}\right\}$ with $n_{j}$ by the mapping $\mathcal{M}$. As an illustration, the relations in Table 3 can be expressed as

$$
\begin{aligned}
& \mathcal{M}(0, \ldots, 0)=1, \\
& \mathcal{M}(1,0, \ldots, 0)=2 .
\end{aligned}
$$

The constant term $d_{n_{j}}^{j}$ is taken to be the minimum constant term in all combination of $f_{n_{k}}^{k}$ with $\mathcal{M}\left(\left\{n_{k}\right\}\right)=n_{j}$, yielding

$$
d_{n_{j}}^{j}=\min _{\left(\left\{n_{k}\right\} \mid n_{j}=\mathcal{M}\left(\left\{n_{k}\right\}\right)\right.}\left[D_{\left\{n_{k}\right\}}+\sum_{k \in \mathcal{L}_{j} \backslash\{i\}} d_{n_{k}}^{k}\right]-\Delta E_{j} .
$$

Remarkably, the functional recursion of $E^{V}$ in Eq. (9) is now simplified to a recursion of the constant terms in Eqs. (22) and (29).

The physical interpretation of the composite functions $n_{j}=0,1,2$ is revealed by considering the patterns of optimal currents around node $j$. For $n_{j}=0$, no resources are drawn from the ancestor, i.e., $\tilde{y}_{0}=0$. Moreover, the optimal currents drawn from the descendents are $\tilde{y}_{n_{k}^{*}}$, which are non-positive according to Table 3 . This shows that $n_{j}=0$ corresponds to a resource providing state (see Fig. 3(a)). 


\begin{tabular}{|c||c|c|c|c|c|}
\hline $\begin{array}{c}\left\{n_{k}\right\}= \\
\left(n_{1}, n_{2}, \ldots, n_{K-1}\right)\end{array}$ & $\tilde{Y}_{\left\{n_{k}\right\}}=\tilde{y}_{n_{j}}$ & $A_{\left\{n_{k}\right\}}=a_{n_{j}}$ & $D_{\left\{n_{k}\right\}}=D_{n_{j}}$ & $\left(y_{1}^{*}, y_{2}^{*}, \ldots, y_{c-1}^{*}\right)$ & $n_{j}$ \\
\hline \hline$(0,0, \ldots, 0)$ & $-\frac{1}{K}$ & $\frac{K}{2(K-1)}$ & $\frac{1}{2 K}$ & $\left(\frac{1}{K}, \frac{1}{K}, \ldots, \frac{1}{K}\right)$ & 1 \\
\hline$(1,0, \ldots, 0)$ & $-\frac{1}{K-1}$ & $\frac{K^{2}-1}{2\left(K^{2}-K-1\right)}$ & $\frac{(K+1)}{2 K(K-1)}$ & $\left(0, \frac{1}{K-1}, \ldots, \frac{1}{K-1}\right)$ & 2 \\
\hline
\end{tabular}

Table 3. Combinations $\left\{n_{k}\right\}$ from descendents that lead to the composite functions $n_{j}=1,2$ given by Table 2. Permutation of $\left(n_{1}, n_{2}, \ldots, n_{K-1}\right)$ result in the same $n_{j}$.

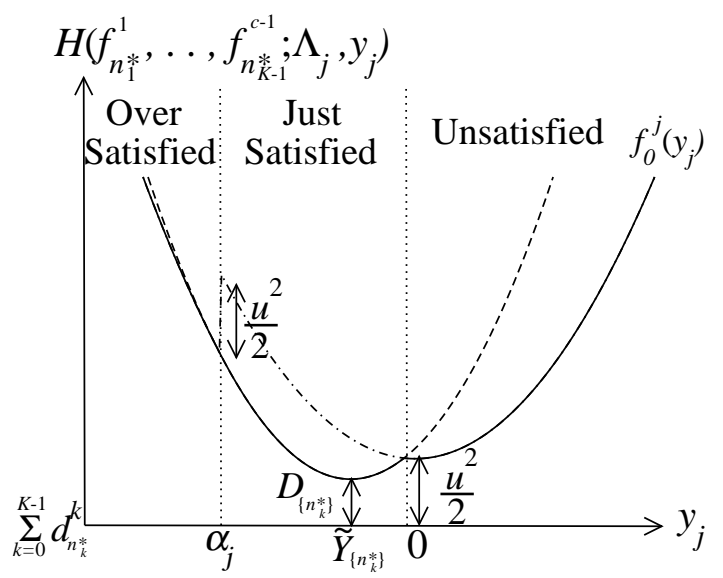

Figure 5. An example of minimization by $\mathcal{H}\left(f_{n_{1}^{*}}^{k=1}, \ldots, f_{n_{K-1}^{*}}^{K-1} ; \Lambda_{j}, y_{j}\right)$ with the combination $\left\{n_{k}\right\}=\left(n_{1}^{*}, n_{2}^{*}, \ldots, n_{K-1}^{*}\right)$. The labels Over Satisfied, Just Satisfied, and Unsatisfied refer to the conditions $\xi_{j}>0, \xi_{j}=0$ and $\xi_{j}<0$ respectively. The just-satisfied portion of $\mathcal{H}$ intersects with $f_{0}^{j}$ at $y_{j}=\alpha_{j}$ as shown.

For $n_{j}=1$, currents of $K^{-1}$ are drawn from the ancestor and all descendents, i.e., $y_{k=1}^{*}=\ldots=y_{K-1}^{*}=K^{-1}$, as shown in Table 3. Hence $n_{j}=1$ corresponds to the singly consuming state. (see Fig. [3(b)).

For $n_{j}=2$, currents of $(K-1)^{-1}$ are drawn from the ancestor and $K-2$ descendents, leaving the link to the remaining descendent idle. This corresponds to the doubly consuming state (see Fig. 3(c)). Nodes with $n_{j}>1$ correspond to other modes of resource consumption.

We return to verify that $E^{V}$ is continuous piecewise quadratic, despite the discontinuity of $f_{0}^{k}$ in Eq. (17). This can be done by substituting $y_{j}=\alpha_{j}$ in Eq. (23) into the composite function Eq. (24) characterized by $A_{\left\{n_{k}^{*}\right\}}, Y_{\left\{n_{k}^{*}\right\}}$ and $D_{\left\{n_{k}^{*}\right\}}$ in Eq. (25). The result shows that $\mathcal{H}=\alpha_{j}^{2} / 2+\sum_{k \in \mathcal{L}_{j} \backslash\{i\}} d_{n_{k}^{*}}^{k}$. Hence it intersects $f_{0}^{j}$ at the lower end of the discontinuity. As shown in Fig. 5, the discontinuity is masked.

We finally derive a simplified expression of $\Delta E_{j}$ from Eq. (10). Since $\mathcal{H}\left(f_{n_{1}}^{1}, \ldots, f_{n_{K-1}}^{K-1} ; \Lambda_{j}, 0\right)$ is already obtained in Eqs. (21) and (24) with $y_{j}=0$,

$$
\Delta E_{j}=\min \left[\min _{\left\{n_{k}\right\}}\left(A_{\left\{n_{k}\right\}} \tilde{Y}_{\left\{n_{k}\right\}}^{2}+D_{\left\{n_{k}\right\}}+\sum_{k \in \mathcal{L}_{j} \backslash\{i\}} d_{n_{k}}^{k}\right),\right.
$$


Optimal Location of Sources in Transportation Networks

$$
\left.\frac{u^{2}}{2} \Theta\left(-\Lambda_{j}\right)+\sum_{k \in \mathcal{L}_{j} \backslash\{i\}} d_{n_{k}}^{k}\right] .
$$

\section{Closed Sets of Cavity Energy Functions: The Intense Simplifications}

\subsection{The Intense Simplification}

In this subsection, we consider networks with no surplus nodes $\left(\phi_{s}=0\right)$. In the singlet regime, the energetically stable configurations consist of only the source nodes and singly consuming nodes. Hence we consider $E^{V}$ with only $f_{0}$ and $f_{1}$ as composite functions. $E^{V}$ is thus given by

$$
E_{k}^{V}\left(y_{k}\right) \doteq\left(d_{0}^{k}, d_{1}^{k}\right),
$$

as a simplification of Eq. (19). Composite functions with $n_{k} \geq 2$ have $d_{n_{k}}^{k}>d_{n_{k}^{*}}^{k}$ in this regime, and their corresponding configurations are not stable. The recursion relations of $d_{0}$ and $d_{1}$ in Eqs. (22) and (29) are simplified to

$$
\begin{aligned}
& d_{0}^{j}=\frac{u^{2}}{2}+\sum_{k \in \mathcal{L}_{j} \backslash\{i\}} \min \left(d_{0}^{k}, d_{1}^{k}\right)-\Delta E_{j}, \\
& d_{1}^{j}=\frac{1}{2 K}+\sum_{k \in \mathcal{L}_{j} \backslash\{i\}} d_{0}^{k}-\Delta E_{j},
\end{aligned}
$$

where

$$
\Delta E_{j}=\min \left[\frac{u^{2}}{2}+\sum_{k \in \mathcal{L}_{j} \backslash\{i\}} \min \left(d_{0}^{k}, d_{1}^{k}\right), \frac{1}{2(K-1)}+\sum_{k \in \mathcal{L}_{j} \backslash\{i\}} d_{0}^{k}\right] .
$$

To determine the pattern of current flow, it is sufficient to consider the recursion of $\epsilon_{j} \equiv d_{1}^{j}-d_{0}^{j}$, given by

$$
\epsilon_{j}=-\gamma-\sum_{k \in \mathcal{\mathcal { L } _ { j }} \backslash\{i\}} \min \left(0, \epsilon_{k}\right),
$$

where

$$
\gamma \equiv \frac{u^{2}}{2}-\frac{1}{2 K} .
$$

The simple recursion leads to a closed set of $K$ cavity energy functions

$$
E_{q}^{V}(y) \doteq\left(d_{0}^{j}, d_{0}^{j}+q \gamma\right) \quad \text { with } q=-1,0, \ldots, K-2 .
$$

These functions are classified to be consuming for $q=-1$, bistable for $q=0$, and resource providing for $q=1, \ldots, K-2$. Their absolute minima are located at $y=-K^{-1}$, both

$y=0$ and $-K^{-1}$, and $y=0$ respectively. We call their states $c$ state, $b$ state and $s$ state respectively; examples for the case of $K=3$ are shown in Fig. 6. The $b$ state behaves in the same way as the $s$ state in the recursion relation, but physically they correspond to different cavity states. The differentiation between $b$ and $s$ states is required only when the entropy of the ground state is calculated. For most other purposes, grouping $b$ and $s$ states together further simplifies the analyses. We thus denote the $b$ and $s$ states as $S$ 

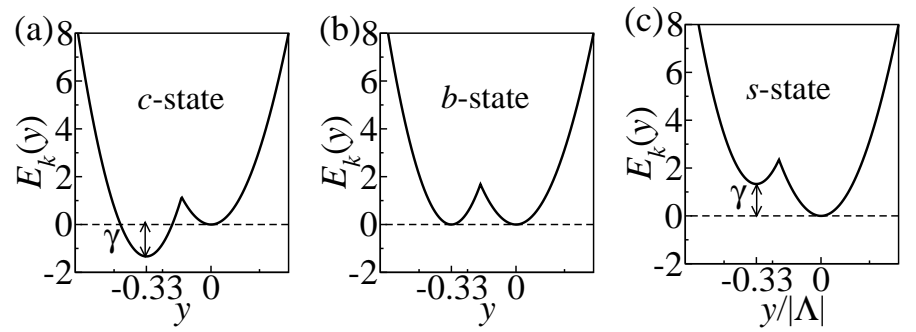

Figure 6. The closed set of $E_{k}(y)$ at $K=3$ and $u^{-1}=5 / 3$, corresponding to (a) the $c$ state $E_{c}^{V}$, (b) the $b$ state $E_{b}^{V}$ and (c) the $s$ state $E_{s}^{V}$.

\begin{tabular}{|c|c|c|c|c|}
\hline Descendent states & $\Delta E_{j}$ & $\mathcal{E}_{j}$ & \multicolumn{2}{|c|}{ Vertex state } \\
\hline \hline$(S, \ldots, S)$ & $\frac{u^{2}}{2}$ & $-\gamma$ & $c$ & $C$ \\
\hline$(C, S, \ldots, S)$ & $\frac{u^{2}}{2}-\gamma$ & 0 & $b$ & $S$ \\
\hline$(C, C, S, \ldots, S)$ & $\frac{u^{2}}{2}-2 \gamma$ & $\gamma$ & $s$ & $S$ \\
$\vdots$ & $\vdots$ & $\vdots$ & $\vdots$ & $\vdots$ \\
$(C, \ldots, C)$ & $\frac{u^{2}}{2}-(K-1) \gamma$ & $(K-2) \gamma$ & $s$ & $S$ \\
\hline
\end{tabular}

Table 4. The recursion relations given by Eq. (35). The column $\Delta E_{j}$ is valid for $u^{-1} \geq \sqrt{K-1}$ in the singlet regime; a constant $(K-1)\left[u^{2} / 2-1 / 2(K-1)\right]$ has to be added to $\Delta E_{j}$ for $u^{-1}<\sqrt{K-1}$ in the singlet regime.

state, and the $c$ state as $C$ state in subsequent analyses. Their recursion relations are summarized in Table 4. (The source state $(s$ state) in this paper should not be confused with the satisfied state (also denoted as $s$ state) in [14]. In fact, the $s, u, b$ states in [14] have the same cavity energy functions as the $c, s, b$ states in this paper respectively.)

The recursion relation in Table 4 can be summarized by the symbolic equations

$$
\underbrace{S+\ldots+S}_{K-1} \rightarrow C
$$

all other combinations $\rightarrow S$.

To calculate the average energy per node, we can apply the same simplification to $\Delta \mathcal{E}_{\text {node }}$ and $\Delta \mathcal{E}_{\text {link }}$ in Eqs. (12) and (13) respectively. We denote the full states of a node as $\mathcal{C}, \mathcal{B}$ and $\mathcal{S}$, respectively representing the consuming, bistable and resource providing states. They are obtained symbolically via

$$
\begin{aligned}
& \underbrace{S+\ldots+S}_{K} \rightarrow \mathcal{C} \\
& \underbrace{C+S+\ldots+S}_{K} \rightarrow \mathcal{B}
\end{aligned}
$$

all other combinations $\rightarrow \mathcal{S}$. 
The energy changes are

$$
\begin{aligned}
\Delta \mathcal{E}_{\text {node }} & =\frac{u^{2}}{2}+\min \left[-\gamma, \sum_{j=1}^{K} \min \left(0, \epsilon_{j}\right)\right], \\
\Delta \mathcal{E}_{\text {link }} & =\min \left(0, \epsilon_{L}, \epsilon_{R}\right) .
\end{aligned}
$$

These expressions are valid for $u^{-1} \geq \sqrt{K-1}$ in the singlet regime; $N_{c}\left[u^{2} / 2-1 / 2(K-1)\right]$ has to be added to the expressions for $u^{-1}<\sqrt{K-1}$ in the singlet regime, where $N_{c}$ is the number of vertices in the $C$ state .

Numerical iterations of Eq. (35) starting from random $\epsilon_{k}$ show that the closed set of $E^{V}$ is stable. The closed set corresponds to the integer cavity fields in the language of the cavity approach. Another example of a closed set of $E^{V}$ is found in the doublet regime as described in Appendix.

We return to discuss the range of the singlet regime. From Eq. (35), it becomes apparent that when $\gamma<0$, we would have $\epsilon_{j}$ always positive, implying that the singly consuming state is always unstable. Hence a necessary condition of the singlet regime is $\gamma \geq 0$, or $u^{-1}<\sqrt{K}$.

Similarly, in the doublet regime, we obtain the recursion relations

$$
\begin{aligned}
& d_{1}^{j}-d_{0}^{j}=-\gamma-\sum_{k \in \mathcal{\mathcal { L } _ { j }} \backslash\{i\}} \min \left(0, d_{1}^{k}-d_{0}^{k}, d_{1}^{k}-d_{0}^{k}\right), \\
& d_{2}^{j}-d_{0}^{j}=-\kappa+\min _{\{k\}}\left(d_{1}^{k}-d_{0}^{k}\right)-\sum_{k \in \mathcal{L}_{j} \backslash\{i\}} \min \left(0, d_{1}^{k}-d_{0}^{k}, d_{1}^{k}-d_{0}^{k}\right),
\end{aligned}
$$

where

$$
\kappa \equiv \frac{u^{2}}{2}-\frac{K+1}{2 K(K-1)} .
$$

Consider the difference $d_{2}^{j}-d_{1}^{j}=-\kappa+\min \left(d_{1}^{k}-d_{0}^{k}\right)+\gamma$. In the singlet regime described by Eq. (35), $d_{2}^{j}-d_{1}^{j}$ is always positive only if $\kappa<0$, implying that $u^{-1}>\sqrt{K(K-1) /(K+1)}$. Combining the two results, the range of the singlet regime is $\sqrt{K(K-1) /(K+1)}<u^{-1}<\sqrt{K}$, agreeing with the result reported in Fig. 2.

It is convenient to represent the recursion relations in the probabilistic framework of BP algorithm [29]. We denote as $\psi_{c}^{j \rightarrow i}$ the probability that node $j$ is in the $C$ state, in the absence of the ancestor node $i$. The probability that node $j$ is in the $S$ state in the absence of $i$ is described as $\psi_{s}^{j \rightarrow i}$. We call $\psi_{c}^{j \rightarrow i}$ and $\psi_{s}^{j \rightarrow i}$ the cavity probabilities. In this framework, Eq. (38a) in the singlet regime can be written as

$$
\psi_{c}^{j \rightarrow i}=\prod_{k \in \mathcal{L}_{j} \backslash\{i\}}\left(1-\psi_{c}^{k \rightarrow j}\right),
$$

and $\psi_{s}^{j \rightarrow i}+\psi_{s}^{j \rightarrow i}=1$. Note that the recursion relation (5) with the functional form of $E^{V}$ is now represented by a recursion of a single scalar $\psi_{c}^{j \rightarrow i}$, greatly simplifying the analysis. This framework will be useful in establishing a connection with the RSB ansatz. 


\subsection{Networks with Surplus Nodes}

In this subsection, we consider networks with surplus nodes $\left(\phi_{s}>0\right)$ in the singlet regime. The recursion relations of the deficient nodes follow those in Table 4, whereas the $E^{V}$ of the surplus nodes are always in the $s$ state. The symbolic equations Eq. (38a) are thus extended to

$$
\begin{aligned}
& \underbrace{S+\ldots+S}_{K-1} \stackrel{\Lambda=-1}{\rightarrow} C . \\
& \text { all other combinations } \stackrel{\Lambda=-1}{\rightarrow} S . \\
& \text { all combinations } \stackrel{\Lambda=A}{\rightarrow} S .
\end{aligned}
$$

The recursion of $\psi_{c}^{j \rightarrow i}$ is given by

$$
\psi_{c}^{j \rightarrow i}=\delta_{\Lambda_{j},-1} \prod_{k \in \mathcal{L}_{j} \backslash\{i\}}\left(1-\psi_{c}^{k \rightarrow j}\right),
$$

and $\psi_{c}^{j \rightarrow i}+\psi_{s}^{j \rightarrow i}=1$.

The full energy change is given by

$$
\Delta \mathcal{E}_{\text {node }}^{i}= \begin{cases}\frac{u^{2}}{2}+\min \left[-\gamma, \sum_{j \in \mathcal{L}_{i}} \min \left(0, \epsilon_{1}^{j}\right)\right], & \Lambda_{i}=-1 \\ \sum_{j \in \mathcal{L}_{i}} \min \left(0, \epsilon_{1}^{j}\right), & \Lambda_{i}=A\end{cases}
$$

and $\Delta \mathcal{E}_{\text {link }}$ is still given by Eq. (40). For deficient nodes, the optimized state is either consuming, bistable, or resource providing. Surplus nodes are fixed to be source nodes. $\Delta \mathcal{E}_{\text {node }}$ and the full states of node $i$ are shown in Table 5 with the corresponding combination of descendent states. The full states of the node $i$ are thus described by the probabilities $\psi_{\mathcal{C}}^{i}, \psi_{\mathcal{B}}^{i}$ and $\psi_{\mathcal{S}}^{i}$ given by

$$
\begin{aligned}
& \psi_{\mathcal{C}}^{i}=\delta_{\Lambda_{i},-1} \prod_{j \in \mathcal{L}_{i}}\left(1-\psi_{c}^{j \rightarrow i}\right), \\
& \psi_{\mathcal{B}}^{i}=\delta_{\Lambda_{i},-1} \sum_{j \in \mathcal{L}_{i}} \psi_{c}^{j \rightarrow i} \prod_{k \in \mathcal{L}_{j} \backslash\{i\}}\left(1-\psi_{c}^{k \rightarrow i}\right),
\end{aligned}
$$

with $\psi_{\mathcal{C}}^{i}+\psi_{\mathcal{B}}^{i}+\psi_{\mathcal{S}}^{i}=1$.

We derive the fraction of nodes with different full states by assuming the independence between descendent branches of a tree. From Eq. (45), the average probability $\left\langle\psi_{c}\right\rangle$ of a node in the cavity $C$ state is thus given by

$$
\left\langle\psi_{c}\right\rangle=\phi_{d}\left[1-\left\langle\psi_{c}\right\rangle\right]^{K-1}
$$

where $\langle\ldots\rangle$ represents averaging over nodes. Iteration of Eq. (45) on Cayley trees reveals that $\left\langle\psi_{c}\right\rangle$ does not approach the stable fixed point of Eq. (50) when $\phi_{d}$ is high. At $\phi_{d}=1$, a period-two solution of $\left\langle\psi_{c}\right\rangle=0$ and 1 emerges. Physically, this corresponds

to alternating layers of consumer and source nodes on Caylay trees. This happens in similar problems such as the Bethe glass [23] and is referred to as the modulation mode. On real networks, nodes are randomly connected, rendering whole layers of consumer 


\begin{tabular}{|c|c|c|c|c|}
\hline \multirow{2}{*}{ Descendent states } & \multicolumn{2}{|c|}{$\Lambda_{i}=-1$} & \multicolumn{2}{c|}{$\Lambda_{i}=A$} \\
\cline { 2 - 5 } & $\Delta \mathcal{E}_{\text {node }}^{j}$ & Full state & $\Delta \mathcal{E}_{\text {node }}^{j}$ & Full state \\
\hline \hline$(S, \ldots, S)$ & $\frac{u^{2}}{2}-\gamma$ & $\mathcal{C}$ & 0 & $\mathcal{S}$ \\
\hline$(C, S, \ldots, S)$ & $\frac{u^{2}}{2}-\gamma$ & $\mathcal{B}$ & $-\gamma$ & $\mathcal{S}$ \\
\hline$(C, C, S, \ldots, S)$ & $\frac{u^{2}}{2}-2 \gamma$ & $\mathcal{S}$ & $-2 \gamma$ & $\mathcal{S}$ \\
$\vdots$ & $\vdots$ & $\vdots$ & $\vdots$ & $\vdots$ \\
$(C, \ldots, C)$ & $\frac{u^{2}}{2}-K \gamma$ & $\mathcal{S}$ & $-K \gamma$ & $\mathcal{S}$ \\
\hline
\end{tabular}

Table 5. $\Delta \mathcal{E}_{\text {node }}$ as given by Eq. (48).

\begin{tabular}{|c|c|}
\hline Vertex states & $\Delta \mathcal{E}_{\text {link }}$ \\
\hline \hline$(S, S)$ & 0 \\
\hline$(C, S)$ & $-\gamma$ \\
\hline$(C, C)$ & $-\gamma$ \\
\hline
\end{tabular}

Table 6. $\Delta \mathcal{E}_{\text {link }}$ as given by Eq. (13).

and source nodes highly unlikely. The period-two situations are suppressed, making a fixed point solution of Eq. (50) possible in random networks. The fraction of nodes with full states $\mathcal{C}, \mathcal{B}$ and $\mathcal{S}$ are thus given by

$$
\begin{aligned}
& f_{\mathcal{C}}=\phi_{d}\left(1-\left\langle\psi_{c}\right\rangle\right)^{K}, \\
& f_{\mathcal{B}}=K \phi_{d}\left\langle\psi_{c}\right\rangle\left(1-\left\langle\psi_{c}\right\rangle\right)^{K-1},
\end{aligned}
$$

with $f_{\mathcal{C}}+f_{\mathcal{B}}+f_{\mathcal{S}}=1$. We leave the discussion of their physical interpretation to Section 5.1 .

\section{The Average Energy}

We evaluate the average energy by considering the energy change due to the addition of new nodes and links. Summarizing Table 5, $\left\langle\Delta \mathcal{E}_{\text {node }}\right\rangle$ becomes

$$
\left\langle\Delta \mathcal{E}_{\text {node }}\right\rangle=\phi_{d}\left[\frac{u^{2}}{2}-\left(1-\left\langle\psi_{c}\right\rangle\right)^{K} \gamma\right]-K\left\langle\psi_{c}\right\rangle \gamma
$$

Similarly, $\left\langle\Delta \mathcal{E}_{\text {link }}\right\rangle$ is given in Table 6 by

$$
\left\langle\Delta \mathcal{E}_{\text {link }}\right\rangle=-\gamma\left[2\left\langle\psi_{c}\right\rangle-\left\langle\psi_{c}\right\rangle^{2}\right]
$$

After some algebra, Eqs. (14), (50) (52) and (53) lead to

$$
\mathcal{E}_{\mathrm{RS}}=\langle\Delta \mathcal{E}\rangle=\phi_{d} \frac{u^{2}}{2}-\left[\left\langle\psi_{c}\right\rangle+\left(\frac{K}{2}-1\right)\left\langle\psi_{c}\right\rangle^{2}\right] \gamma .
$$

When a deficient node changes from a source to a consumer, the energy of the node reduces by $\gamma$ from $u^{2} / 2$. We thus identify the coefficient of $\gamma$ in Eq. (54) to be the 


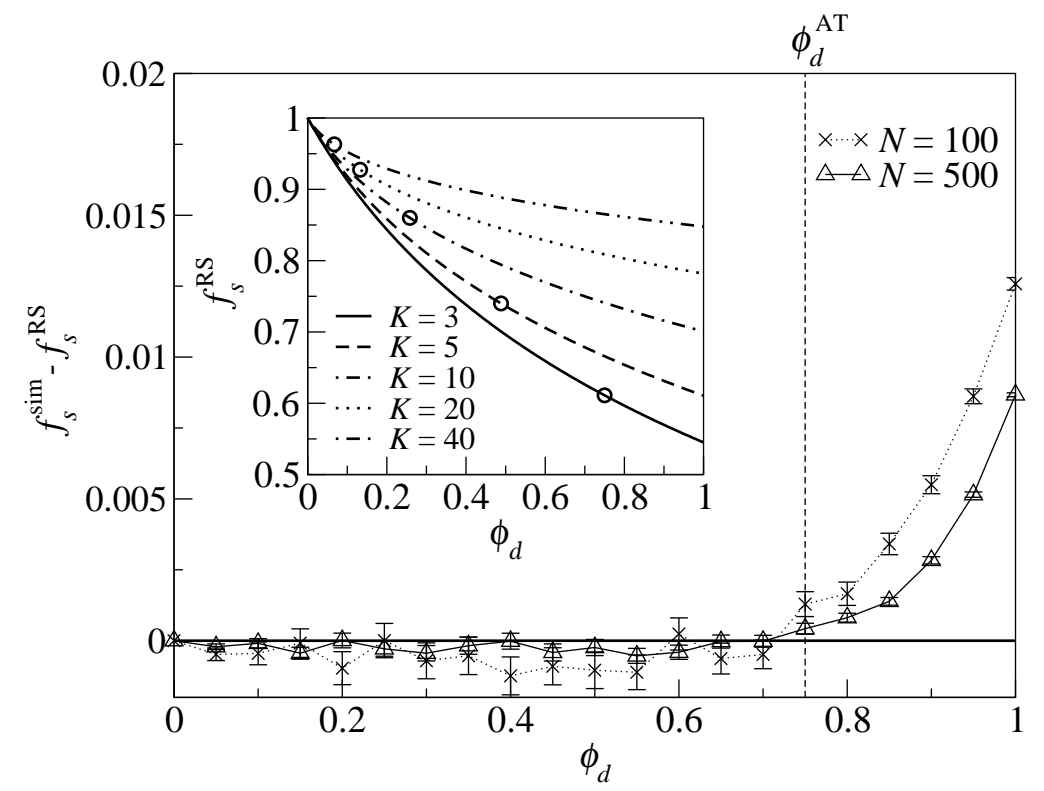

Figure 7. The average fraction of source nodes in simulations with $K=3$, $N=100,500$ and $3000 N$ steps with $N_{\text {flip }}=4$, as compared with the $f_{s}^{\mathrm{RS}}$ obtained from the RS ansatz. Inset: $f_{s}^{\mathrm{RS}}$ for different values of $K$. The circles indicate the values of $\phi_{d}$ above which the RS assumption is not stable.

fraction of consumer nodes. The fraction $f_{s}^{\mathrm{RS}}$ of source nodes is then given by

$$
f_{s}^{\mathrm{RS}}=1-\left\langle\psi_{c}\right\rangle-\left(\frac{K}{2}-1\right)\left\langle\psi_{c}\right\rangle^{2} .
$$

Note that $f_{s}^{\mathrm{RS}}$ is distinguished from $f_{\mathcal{S}}^{\mathrm{RS}}$, since $f_{s}=f_{\mathcal{S}}+f_{\mathcal{B}} / 2$, i.e., $f_{s}$ also counts those bistable nodes that become source nodes in the network configuration. Through the linear relationship $\langle\mathcal{E}\rangle=\phi_{d} u^{2} / 2-f_{c}^{\mathrm{RS}} \gamma=1 / 2 K-\left(1-\phi_{d}\right) u^{2} / 2+f_{s}^{\mathrm{RS}} \gamma$, we consider $f_{s}$ as a measure of the average energy $\langle\mathcal{E}\rangle$.

The inset of Fig. 7 shows the fraction of source nodes as a function of $\phi_{d}$ derived from Eqs. (50) and (55). For all connectivities $K, f_{s}^{\mathrm{RS}}$ decreases with $\phi_{d}$. A higher connectivity leads to an increase in $f_{s}^{\mathrm{RS}}$ since more nodes are required to convert to source nodes to satisfy the demand of a consumer node.

Figure 7 shows the difference between $f_{s}^{\mathrm{RS}}$ and $f_{s}^{\text {sim }}$ obtained from numerical simulations, in which the energy of real instances is minimized by the GSAT algorithm as described in Section 2.2, The differences between $f_{s}^{\mathrm{RS}}$ and $f_{s}^{\text {sim }}$ are roughly zero when $\phi_{d}$ is below some critical value. Above the critical value, $f_{s}^{\mathrm{RS}}$ is significantly lower than $f_{s}^{\text {sim }}$. Hence the energy $\mathcal{E}_{\mathrm{RS}}$ is lower than the simulated energy. This discrepancy is related to the instability of the RS ansatz, which will be discussed below.

\subsection{The Soft Nodes in the Ground States}

Nodes with the full state $\mathcal{B}$ are bistable between the consumer and source states. Figure 8 shows an example with two central nodes in the $\mathcal{B}$-state. Using Table 4 , the cavity energy functions passed among them are worked out. In particular, $E_{b}^{V}$ is 
(a)

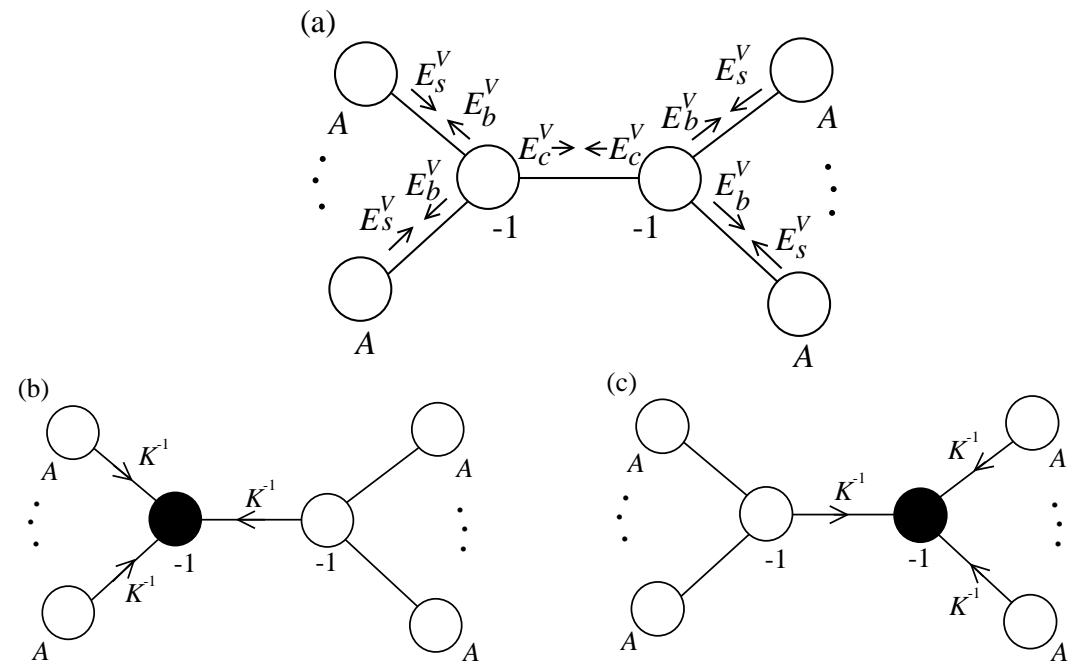

Figure 8. (a) An example of a configuration with two central soft nodes, annotated with cavity energy functions passed among them. (b-c) The two degenerate states of configuration in (a).

sent from the bistable nodes to all its resource-providing neighbors, implying a zero current or a current of $-K^{-1}$ would have no effect on the optimized energy. Hence, the central pairs correspond to the soft nodes which can be a consumer or a source node in different degenerate configurations. These degenerate configurations are connected in the configuration space, since transitions among them only involve the flipping of states of the soft nodes. The RS ansatz assumes that the configuration space is dominated by a single cluster whose states can be obtained from each other by flipping the states of the soft nodes [30]. In other words, using the notions of $\mathcal{S}, \mathcal{C}$ and $\mathcal{B}$ states, the single cluster is described by a unique set of $\mathcal{S}, \mathcal{C}$ and $\mathcal{B}$ labels for each node. On the other hand, if the network enters the RSB phase, the configuration space is dominated by numerous clusters, each with its own set of $\mathcal{S}, \mathcal{C}$ and $\mathcal{B}$ states of the nodes. Transitions among the clusters involve flipping the hard (non-soft) nodes as well. In this section, we discuss the RS case.

The fraction of soft nodes is given by the fraction of nodes with full state $\mathcal{B}$. From Eq. (51), we have

$$
f_{\text {soft }}^{\mathrm{RS}}=f_{\mathcal{B}}=K \phi_{d}\left\langle\psi_{c}\right\rangle\left(1-\left\langle\psi_{c}\right\rangle\right)^{K-1} .
$$

On the other hand, the hard nodes are either consumer or source nodes in all degenerate states. They correspond to nodes in the backbone in a vertex cover [6, 7, 8]. Surplus nodes are certainly in the source backbone. Deficient nodes can be found either in the consumer or source backbone. From Table 5, the fraction of nodes in the consumer backbone is given in Eq. (51) by the probability of finding a node with full state $\mathcal{C}$, namely,

$$
f_{\text {con-bone }}^{\mathrm{RS}}=f_{\mathcal{C}}=\phi_{d}\left(1-\left\langle\psi_{c}\right\rangle\right)^{K}
$$

Remarkably, it can be shown easily $f_{\text {soft }}^{\mathrm{RS}} / 2+f_{\text {con-bone }}^{\mathrm{RS}}=1-f_{s}^{\mathrm{RS}}$ which implies that 


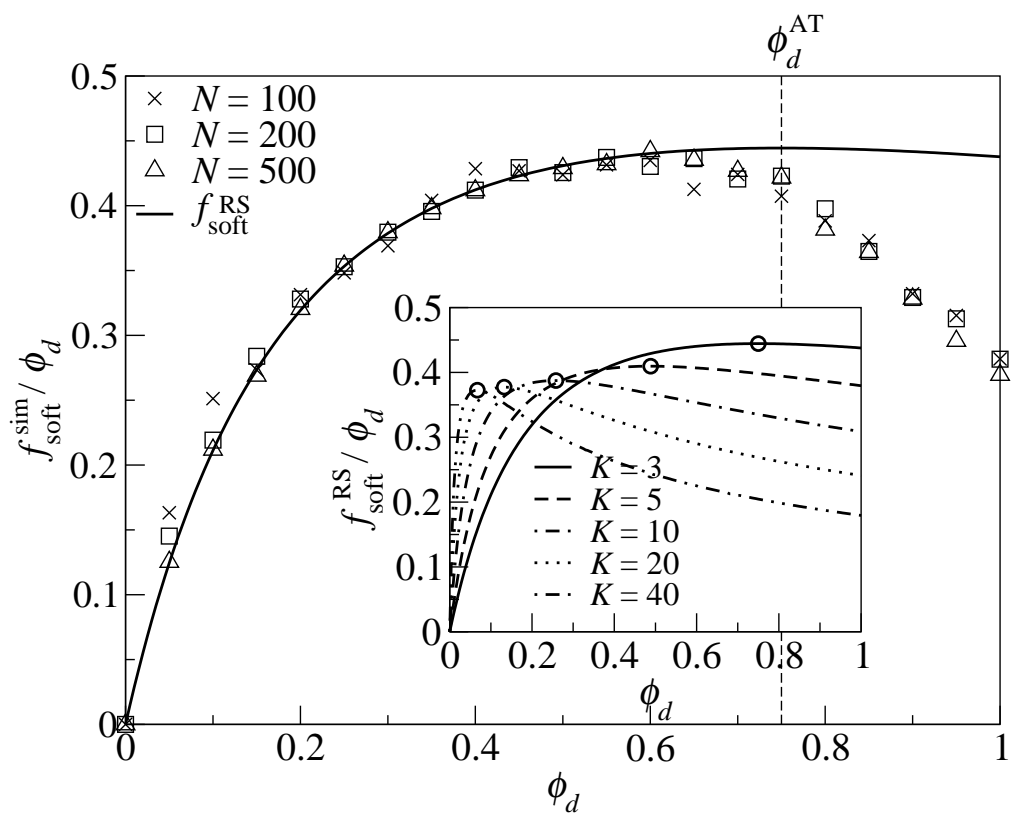

Figure 9. The average fraction of soft nodes in simulations of $K=3$ and $3000 N$ steps with $N_{\text {flip }}=4$, as compared with $f_{\text {soft }}^{\mathrm{RS}}$. Inset: $f_{s}^{\mathrm{RS}} / \phi_{d}$ for different values of $K$. The circles indicates the values of $\phi_{d}$ above which the RS assumption is not stable.

exactly half of the soft nodes are consumers.

The fraction of soft nodes relative to the fraction of deficient nodes obtained from Eq. (56) is shown in the inset of Fig. 9. When the fraction $\phi_{d}$ of deficient nodes increases from 0 , the fraction increases until it reaches a maximum value at $\left\langle\psi_{c}\right\rangle=K^{-1}$ and $\phi_{d}=K^{K-2} /(K-1)^{K-1}$. When $\phi_{d}$ is small, most deficient nodes are surrounded by surplus nodes and hence are found in the consumer backbone, leading to a small fraction of soft nodes. When $\phi_{d}$ increases, the probability of finding contiguous deficient nodes increases, which leads to an increase in $f_{\text {soft }}^{\text {RS }}$.

The fraction of soft nodes in simulations is compared with $f_{\text {soft }}^{\mathrm{RS}}$ in Fig. 9, In simulations, we use an algorithm similar to the GSAT [31] to lower the energy of the system until it becomes steady. Then we allow further cluster flips and identify the nodes which can be flipped with no change in global energy, such as the example in Fig. 8(b) and (c). Similar to Fig. 7, the simulation results have an excellent agreement with the RS result when $\phi_{d}$ is below the value at the peak of $f_{\text {soft }}^{\mathrm{RS}} / \phi_{d}$. Above this critical value, the fraction of soft nodes in simulations is less than the RS prediction, which will be shown to be due to RS instability.

Algorithmically, a high fraction of soft nodes leads to an unfavorable consequence. After the convergence of $\mathrm{BP}$ on real instances, nodes with $\mathcal{B}$ state are bistable and a further determination of the final optimal configuration is required on the resulting sub-graph of $\mathcal{B}$ nodes. A random assignment of $\mathcal{B}$ nodes to either consuming or resourceproviding does not generally result in an optimal configuration. When $\phi_{d}$ is low, the sub-graph of $\mathcal{B}$ nodes are disconnected and the assignment is easy. When $\phi_{d}$ is high, the sub-graphs of $\mathcal{B}$ nodes are connected and the assignment is more difficult. 


\subsection{The Instability of the RS Ansatz}

The discrepancy between the simulation results and the predicted average energy $\mathcal{E}_{\mathrm{RS}}$ and $f_{\text {soft }}^{\mathrm{RS}}$ suggests that the RS ansatz is unstable at high $\phi_{d}$. In the RS formalism, a single ground state is assumed. We thus examine the stability of this assumption against the picture of multiple ground states, by relaxing the constraints of $\psi_{s}^{j \rightarrow i}=0,1$. In other words, $0 \leq \psi_{c}^{j \rightarrow i} \leq 1$. To study the stability of the integer ansatz of $\psi_{c}^{j \rightarrow i}$, we define the variables

$$
\begin{aligned}
& \eta_{c}^{j \rightarrow i}=\delta_{\psi_{c}^{j \rightarrow i}, 1} \\
& \eta_{s}^{j \rightarrow i}=\delta_{\psi_{s}^{j \rightarrow i}, 1}
\end{aligned}
$$

and $\eta_{g}^{j \rightarrow i}=1-\eta_{c}^{j \rightarrow i}-\eta_{s}^{j \rightarrow i}$. Thus, the recursion rule (45) is extended to include noninteger values of $\psi_{c}^{j \rightarrow i}$. $\eta_{g}^{j \rightarrow i}=1$ corresponds to the onset of non-zero probabilities over the range of $0<\psi^{j \rightarrow i}<1$, indicating the occurrence of glassy behavior with node $j$ having a probabilistic distribution of $C$ and $S$ states in the stable states of the configuration space. Hence the RS solution is stable only if $\eta_{g}^{j \rightarrow i}=0$ for all $j \rightarrow i$ is a stable fixed point.

We thus formulate the recursion relations of $\eta_{c}^{j \rightarrow i}, \eta_{s}^{j \rightarrow i}$ and $\eta_{g}^{j \rightarrow i}$ by considering Table 4, namely,

$$
\begin{aligned}
& \eta_{c}^{j \rightarrow i}=\delta_{\Lambda_{j},-1} \prod_{k=1}^{K-1} \eta_{s}^{k \rightarrow j} \\
& \eta_{s}^{j \rightarrow i}=\delta_{\Lambda_{j}, A}+\delta_{\Lambda_{j},-1}\left[1-\prod_{k=1}^{K-1}\left(\eta_{s}^{k \rightarrow j}+\eta_{g}^{k \rightarrow j}\right)\right] \\
& \eta_{g}^{j \rightarrow i}=\delta_{\Lambda_{j},-1}\left[\prod_{k=1}^{K-1}\left(\eta_{s}^{k \rightarrow j}+\eta_{g}^{k \rightarrow j}\right)-\prod_{k=1}^{K-1} \eta_{s}^{k \rightarrow j}\right] .
\end{aligned}
$$

Denoting the site average of $\eta_{c}^{j \rightarrow i}, \eta_{s}^{j \rightarrow i}$ and $\eta_{g}^{j \rightarrow i}$ as $\left\langle\eta_{c}\right\rangle,\left\langle\eta_{s}\right\rangle$ and $\left\langle\eta_{g}\right\rangle$ respectively, we obtain their stable fixed points by solving

$$
\begin{aligned}
& \left\langle\eta_{c}\right\rangle=\phi_{d}\left\langle\eta_{s}\right\rangle^{K-1} \\
& \left\langle\eta_{s}\right\rangle=1-\phi_{d}\left(\left\langle\eta_{s}\right\rangle+\left\langle\eta_{g}\right\rangle\right)^{K-1} \\
& \left\langle\eta_{g}\right\rangle=\phi_{d}\left[\left(\left\langle\eta_{s}\right\rangle+\left\langle\eta_{g}\right\rangle\right)^{K-1}-\left\langle\eta_{s}\right\rangle^{K-1}\right]
\end{aligned}
$$

For all $\phi_{d},\left\langle\eta_{g}\right\rangle=0$ is a trivial solution of the last line of Eq. (60), and the above recursions reduce to the RS recursions (45). By introducing a small perturbation $\delta \eta_{g}^{k \rightarrow j}$ to $\eta_{g}^{k \rightarrow j}=0$, we obtain the corresponding $\delta \eta_{g}^{j \rightarrow i}$. The solution of $\left\langle\eta_{g}\right\rangle=0$ is stable under the perturbation if

$$
\left|\frac{\left\langle\delta \eta_{g}^{j \rightarrow i}\right\rangle}{\left\langle\delta \eta_{g}^{k \rightarrow j}\right\rangle}\right|=(K-1) \phi_{d}\left\langle\eta_{s}\right\rangle^{K-2} \leq 1,
$$

Alternatively, the stability of the RS solution can be studied by considering the propagation of fluctuations $\left\langle\left(\delta \psi_{c}^{k \rightarrow j}\right)^{2}\right\rangle$ under the recursion relation Eq. (45) [34]. This 

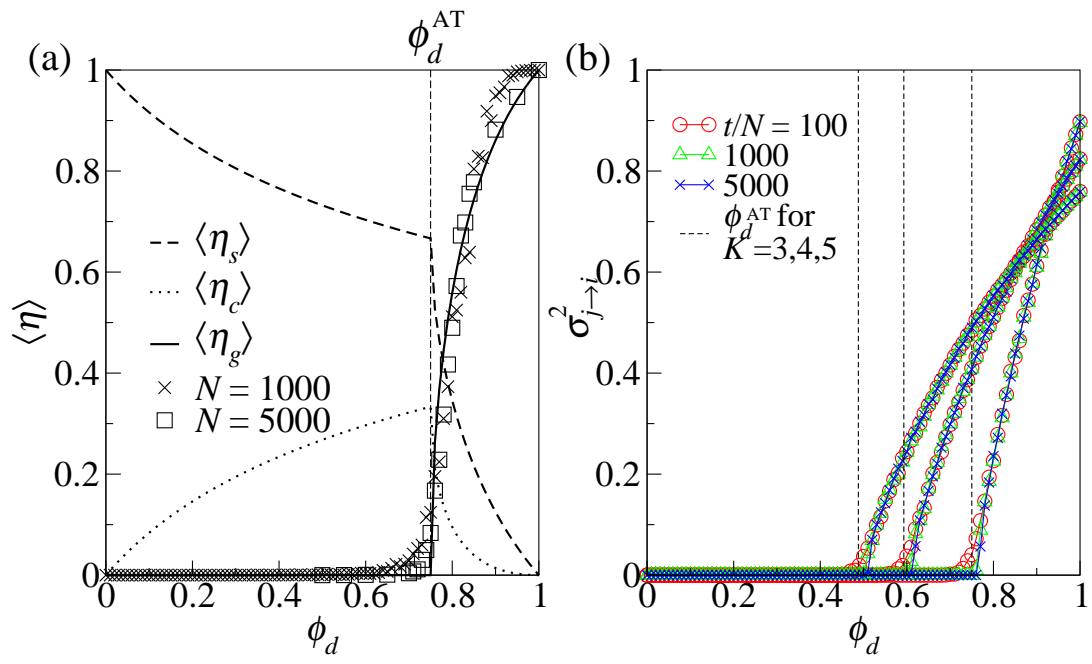

Figure 10. (a) The stable solution of Eq. (60) for $K=3$. The symbols represent the simulated fraction of non-converging BP messages. (b) The dependence of the variance $\sigma_{j \rightarrow i}^{2}$ on $\phi_{d}$. The dotted lines indicate the corresponding values of $\phi_{d}^{\mathrm{AT}}$.

leads to the Almeida-Thouless (AT) stability condition,

$$
\frac{\left\langle\left(\delta \psi_{c}^{j \rightarrow i}\right)^{2}\right\rangle}{\left\langle\left(\delta \psi_{c}^{k \rightarrow j}\right)^{2}\right\rangle}=(K-1) \phi_{d}\left\langle\left(\psi_{s}\right)^{2}\right\rangle^{K-2} \leq 1 .
$$

In the RS regime, $\left\langle\left(\psi_{s}\right)^{2}\right\rangle=\left\langle\eta_{s}\right\rangle$ since $\psi_{s}^{j \rightarrow i}=0$ or 1 . The AT stability condition is thus equivalent to Eq. (61).

The stable solution of $\left\langle\eta_{c}\right\rangle,\left\langle\eta_{s}\right\rangle$ and $\left\langle\eta_{g}\right\rangle$ for $K=3$ is shown in Fig. 10(a). The RS solution becomes unstable when $\phi_{d}>0.75$ for $K=3$. This critical value agrees with those found in the simulation of the average energy and the fraction of soft nodes. For general values of $K$, simple algebra leads to the AT line

$$
\phi_{d}^{\mathrm{AT}}=\frac{K^{K-2}}{(K-1)^{K-1}}
$$

which separates the RS and the RSB phases in the space as shown in the inset of Fig. 11. From Eqs. (50), (56) and ([62), it can be shown that $\left\langle\psi_{s}\right\rangle=f_{\text {soft }}^{\mathrm{RS}}=K^{-1}$ on the AT line.

In the large $K$ limit, $\phi_{d}^{\mathrm{AT}}$ approaches $e / K$. This result has an interesting connection with the vertex cover problem. Considering the covered set as the set of source nodes, all links involving surplus nodes are covered. The remaining links are those among the deficient nodes. These deficient nodes have at least one neighbor being a deficient node. Hence from Table 5, their surplus node neighbors do not affect the states of the deficient nodes. Rather, their states are determined by the states of their deficient node neighbors. Thus, the problem of minimizing the covered set size reduces to one that minimizes the subset size of covered nodes in the subnetwork of deficient nodes as sketched in Fig. 11. In the large $K$ limit, this subnetwork has a Poissonian connectivity distribution with a mean $K \phi_{d}$. The result $K \phi_{d}^{\mathrm{AT}}=e$ agrees with the point of $\mathrm{RS}$ instability derived in [6, 7]. 


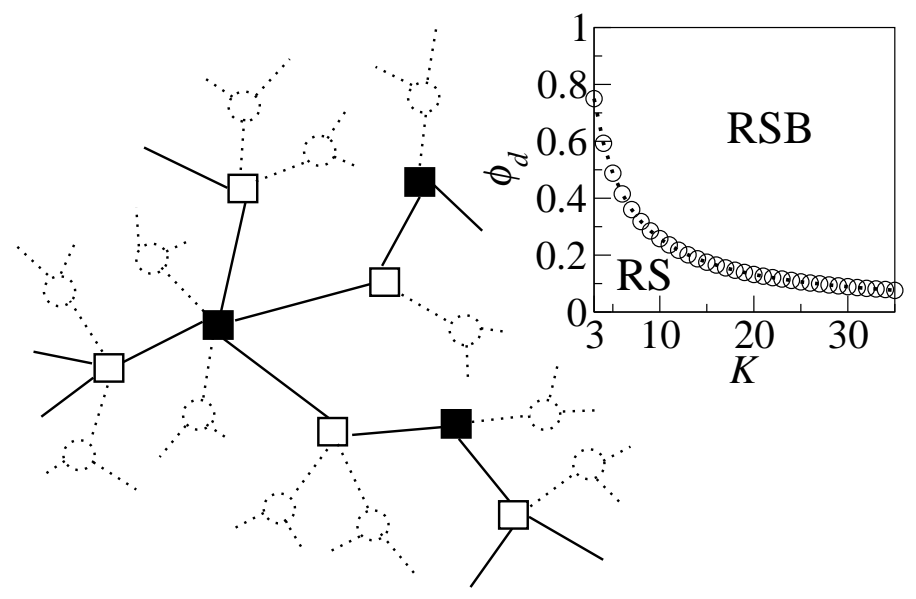

Figure 11. Sketch of the Poissonian subnetwork of deficient nodes in the large connectivity limit with only a fraction $O\left(K^{-1}\right)$ of deficient nodes (squares) and the rest being surplus nodes (circles). Filled and unfilled symbols represent consumer or source states respectively. Inset: The RS and RSB phases in the $K-\phi_{d}$ space.

Comparisons between the simulation results and the RS analytical results from Figs. 7 and 9 have yielded evidence of an AT transition in real instances at $\phi_{d}=\phi_{d}^{\mathrm{AT}}$. Here we provide two more supporting numerical experiments. In the first experiment, we consider the BP algorithm initialized with $\psi_{c}^{j \rightarrow i}=0,1$ for all $j \rightarrow i$. As shown in Fig. 10(a), effectively all messages converge to their steady states in the RS regime. However, a significant fraction of messages fluctuates between 0 and 1 when $\phi_{d}$ rises above $\phi_{d}^{\mathrm{AT}}$, indicating the breakdown of the RS ansatz. It leads to the non-convergence of the $\mathrm{BP}$ algorithm on real instances. As shown in Fig. 10(a), the fraction of non-converging BP messages has an excellent agreement with the theoretical values of $\left\langle\eta_{g}\right\rangle$. Consequently, improved algorithms are needed for ground state searching. Algorithmically, decimation procedures, such as those used in the survey propagation (SP) algorithm [4], are required. We have tested the BP algorithm with decimation and obtained results with simulated energy lower than the GSAT algorithm, which will be reported elsewhere.

In the second experiment, we consider numerical iterations of Eq. (45) using population dynamics [9, 10]. We start with different sets of initial values of $\psi_{c}^{j \rightarrow i}=0,1$, each set following the same sequence of random connections among the nodes. When the dynamics reaches the steady state, we measure the variance $\sigma_{j \rightarrow i}^{2}=\overline{\left(\psi_{c}^{j \rightarrow i}\right)^{2}}-\left(\overline{\psi_{c}^{j \rightarrow i}}\right)^{2}$, where the overline denotes the average over random initial conditions. $\sigma_{j \rightarrow i} \approx 0$ implies that the cavity state of node $j$ is independent of the boundary condition of the tree represented by the population dynamics, and is always frozen in either $S$ or $C$ states. $\sigma_{j \rightarrow i}>0$ implies that the cavity state of node $j$ is dependent on the boundary conditions and shows a long range correlation. The numerical results of $\sigma_{j \rightarrow i}$ averaged over nodes are shown in Fig. 10(b) for $K=3,4,5$. In the figure $\left\langle\sigma_{j \rightarrow i}\right\rangle>0$ when $\phi_{d}>\phi_{d}^{\mathrm{AT}}$ with almost no dependence on the number of iterations in the population dynamics. 


\section{The One-step Replica Symmetry Breaking Ansatz}

\subsection{The 1RSB Formalism}

In the RSB formalism, the network behavior is explained by the dominance of numerous states in the configuration space, instead of a single ground state in the RS formalism. Here, we consider the 1RSB ansatz [32, 33] where the density of states with energy $\mathrm{e}=\mathcal{E} / N$ per node is assumed to have the form $\mathcal{N}_{N}(\mathrm{e})=\exp [N \Sigma(\mathrm{e})]$, for a network of $N$ nodes and total energy $\mathcal{E} . \Sigma(\mathrm{e})$ is called the complexity or configurational entropy. For small changes in the average energy, we can write $\Sigma(\mathrm{e})=x\left(\mathrm{e}-\mathrm{e}^{R}\right)$ where $\mathrm{e}^{R}$ is the reference energy. This assumption leads to a recursion for the cavity probability functional $P_{j}\left[E_{j}^{V}\right]$

$$
\begin{aligned}
& P_{j}\left[E_{j}^{V}\left(y_{j}\right)\right]=\frac{1}{\Xi} \prod_{k \in \mathcal{L}_{j} \backslash\{i\}}\left(\int \mathcal{D} E_{k}^{V}\left(y_{k}\right) P_{k}\left[E_{k}^{V}\left(y_{k}\right)\right]\right) \\
& \times \prod_{y_{j}}\left(\delta\left[E_{j}^{V}\left(y_{j}\right)-\mathcal{H}\left(E_{1}^{V}, \ldots, E_{K-1}^{V} ; \Lambda_{j}, y_{j}\right)+\Delta E_{j}\left(E_{1}^{V}, \ldots, E_{K-1}^{V} ; \Lambda_{j}\right)\right]\right. \\
& \left.\times \exp \left[-x \Delta E_{j}\left(E_{1}^{V}, \ldots, E_{K-1}^{V} ; \Lambda_{j}\right)\right]\right)
\end{aligned}
$$

where $\Xi$ is the normalization constant. We now define the right hand side of Eq. (64) to be $\mathcal{H}_{P}$ and the recursion can be represented by $P_{j}\left[E_{j}^{V}\right]=\mathcal{H}_{P}\left(P_{k=1}, \ldots, P_{c-1}, E_{j}^{V} ; \Lambda_{j}, x\right)$. Solving the recursion in Eq. (64) by population dynamics is equivalent to solving for a stable functional distribution $\mathcal{Q}$ in

$$
\begin{aligned}
& \mathcal{Q}\left[P_{j}\right]=\int d \Lambda_{j} \rho\left(\Lambda_{j}\right) \prod_{k=1}^{K-1} \int \mathcal{D} P_{k} \mathcal{Q}\left[P_{k}\right] \\
& \times \prod_{E_{j}^{V}} \delta\left\{P_{j}\left[E_{j}^{V}\right]-\mathcal{H}_{P}\left(P_{k=1}, \ldots, P_{c-1}, E_{j}^{V} ; \Lambda_{j}, x\right)\right\},
\end{aligned}
$$

which is an analogy to the RS case of solving for $\mathcal{P}\left[E^{V}\right]$ in Eq. (11). Note that the RS recursions in Eq. (9) correspond to the recursions of the cavity energy functions $E^{V}$ yielding a solution of the functional $P\left[E^{V}\right]$, while the 1RSB recursions in Eq. (64) correspond to recursions of the functional probability $P\left[E^{V}\right]$ yielding a solution of the probability functional $Q[P]$.

To analyze the physical properties of the network, we write the partition function when $K$ cavity probability functional feeds a central node.

$$
\Xi_{\text {node }}=\prod_{j \in \mathcal{L}_{i}}\left(\int \mathcal{D} E_{j}^{V} P_{j}\left[E_{j}^{V}\right]\right) \exp \left[-x \Delta \mathcal{E}_{\text {Node }}\left(E_{1}^{V}, \ldots, E_{K}^{V} ; \Lambda_{i}\right)\right] .
$$

with $\Delta \mathcal{E}_{\text {node }}$ given by Eq. (12). Similarly, the partition function obtained by bridging two trees with a link is

$$
\Xi_{\text {link }}=\int \mathcal{D} E_{L}^{V} \mathcal{D} E_{R}^{V} P_{L}\left[E_{L}^{V}\right] P_{R}\left[E_{R}^{V}\right] \exp \left[-x \Delta \mathcal{E}_{\text {link }}\left(E_{L}^{V}, E_{R}^{V}\right)\right] .
$$

The average configuration free energy is given by

$$
\Phi(x)=-\frac{1}{x}\left(\left\langle\ln \Xi_{\text {node }}\right\rangle-\frac{K}{2}\left\langle\ln \Xi_{\text {link }}\right\rangle\right)
$$


The averages $\langle\ldots\rangle$ are taken over $\Lambda_{i}$ and $P_{j}$ from the distribution $\mathcal{Q}\left[P_{j}\right] . \Phi$ is related to the complexity $\Sigma$ and the average energy e by

$$
x \Phi=x \mathrm{e}-\Sigma,
$$

with $\partial \Sigma / \partial \mathrm{e}=x . \Sigma$ and $\mathrm{e}$ are parametrically dependent on $x$ via

$$
\begin{aligned}
& \Sigma=x^{2} \frac{\partial \Phi}{\partial x}, \\
& \mathrm{e}=\frac{\partial(x \Phi)}{\partial x} .
\end{aligned}
$$

While solving for $P\left[E^{V}\right]$ in Eq. (64) is in general difficult, simple solutions can be obtained if a closed set of countably many $E^{V}$ is sufficient to describe the recursions of $E^{V}$. The singlet regime in which the $S$ and $R$ states form a closed set is a good example. We emphasize, however, that the techniques are generally applicable to regions beyond the singlet regime where closed sets of $E^{V}$ are found, such as the commensurate point in the doublet regime discussed in Appendix A.

\subsection{The 1RSB Solution}

In the singlet regime. there are only two representative states, $C$ and $S$ states, we parametrize $P\left[E^{V}\right]$ as

$$
P_{j}\left[E_{j}^{V}\right]=\psi_{c}^{j} \delta\left(E_{j}^{V}-E_{c}^{V}\right)+\psi_{s}^{j} \delta\left(E_{j}^{V}-E_{s}^{V}\right) .
$$

Using Table 4 to obtain $\Delta E$ for different combinations of $C$ and $S$ states, Eq. (64) can be simplified to

$$
\begin{aligned}
\psi_{c}^{j \rightarrow i} & =\delta_{\Lambda_{j},-1} \frac{e^{-x u^{2} / 2}}{\mathcal{Z}^{j \rightarrow i}} \prod_{k \in \mathcal{L}_{j} \backslash\{i\}} \psi_{s}^{k \rightarrow j}, \\
\psi_{s}^{j \rightarrow i} & =\delta_{\Lambda_{j},-1} \frac{e^{-x u^{2} / 2}}{\mathcal{Z}^{j \rightarrow i}}\left[\prod_{k \in \mathcal{L}_{j} \backslash\{i\}}\left(\psi_{c}^{k \rightarrow j} e^{x \gamma}+\psi_{s}^{k \rightarrow j}\right)-\prod_{k \in \mathcal{L}_{j} \backslash\{i\}} \psi_{s}^{k \rightarrow j}\right]+\delta_{\Lambda_{j}, A}, \\
\mathcal{Z}^{j \rightarrow i} & =e^{-x u^{2} / 2} \prod_{k \in \mathcal{L}_{j} \backslash\{i\}}\left(\psi_{c}^{k \rightarrow j} e^{x \gamma}+\psi_{s}^{k \rightarrow j}\right) .
\end{aligned}
$$

The above recursions of $\psi_{c}^{j \rightarrow i}$ are gross simplifications of the recursions of the functional probabilities in Eq. (64). These equations can be solved by population dynamics involving a pool of values of $\psi_{c}^{j \rightarrow i}$ with $0 \leq \psi_{c}^{j \rightarrow i} \leq 1$. Alternatively, the solution to the 1RSB recursion can be found by directly solving for the distribution $Q\left(\psi_{c}\right)$ in Eq. (65) which is isomorphic to $\mathcal{Q}[P]$ in Eq. (65)).

The stable solution of $Q\left(\psi_{c}\right)$ for $K=3$ and $x=0$ is shown in Fig. 12(a). When $\phi_{d} \leq \phi_{d}^{\mathrm{AT}}$, there are no fractional components of $\psi_{c}$, and $Q\left(\psi_{c}\right)=\left\langle\psi_{c}\right\rangle \delta\left(\psi_{c}-1\right)+(1-$ $\left.\left\langle\psi_{c}\right\rangle\right) \delta\left(\psi_{c}\right)$. When $\phi_{d}>\phi_{d}^{\mathrm{AT}}$, non-zero components of $0<\psi_{c}<1$ exist. This agrees with the result in Section 5.2 that in this regime, the RS solution is an unstable solution of Eq. (65). When $\phi_{d}=1$, there is no disorder in the capacities. All vertices are identical and $Q\left(\psi_{c}\right)=\delta\left(\psi_{c}-\left\langle\psi_{c}\right\rangle\right)$ where $\left\langle\psi_{c}\right\rangle$ is given by the RS equation (50). This means that 

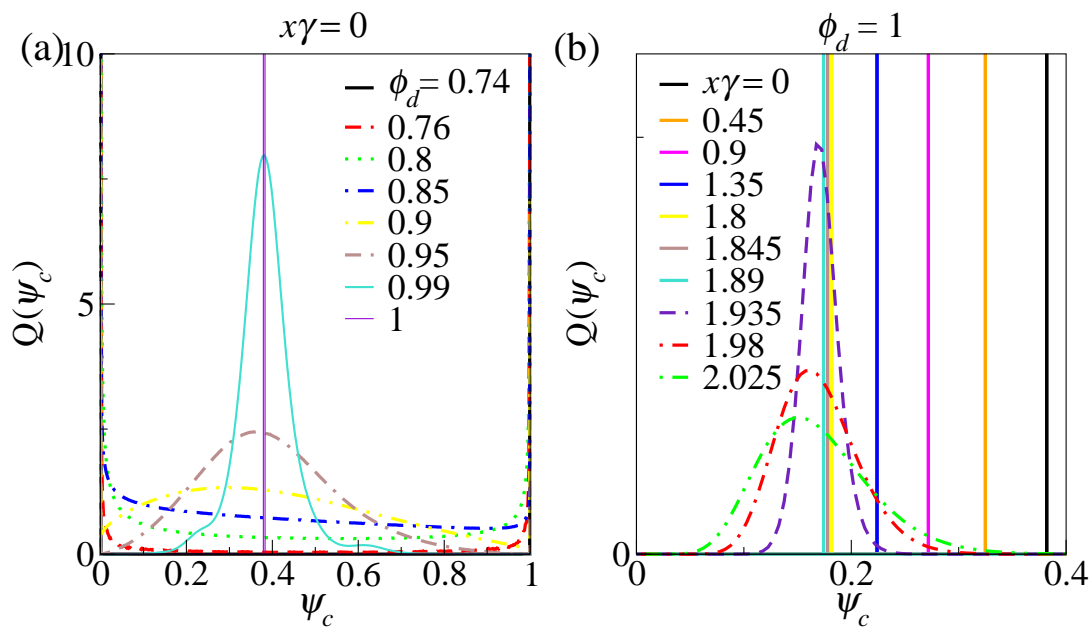

Figure 12. (a) The stable solution of $Q\left(\psi_{c}\right)$ obtained by solving Eq. (65) in the singlet regime using Eq. (73) with $K=3$ and $x=0$. (b) The stable solution of $Q\left(\psi_{c}\right)$ with $K=3$ and $\phi_{d}=1$.

among the different states of the system, all vertices are equally probable to be in $C$ state with probability $\left\langle\psi_{c}\right\rangle$. The stable solutions of $Q\left(\psi_{c}\right)$ are dependent on $x$ via the factor $x \gamma$. In the case of $\phi_{d}=1$, the dependence of $Q\left(\psi_{c}\right)$ on $x$ is shown in Fig. 12(b). The position of the delta peak at $\left\langle\psi_{c}\right\rangle=\left(1-\left\langle\psi_{c}\right\rangle\right)^{K-1} /\left[\left(e^{x \gamma}-1\right)\left\langle\psi_{c}\right\rangle+1\right]^{K-1}$ shifts to left from $x \gamma=0$ to $x \gamma=x^{(\mathrm{I})} \gamma=1.92$. For $x>x^{(\mathrm{I})}$ in Fig. 12(b), $Q\left(\psi_{c}\right)$ becomes a continuous distribution, indicating the instability of the 1RSB ansatz to be discussed in the next subsection.

With the stable solution of $Q\left(\psi_{c}\right)$, we derive the complexity $\Sigma(\mathrm{e})$ in the singlet regime. Obtaining $\Delta \mathcal{E}_{\text {node }}$ in Table 5, we write the partition function $\Xi_{\text {node }}$ in Eq. (66) as

$$
\begin{aligned}
\Xi_{\text {node }}= & \delta_{\Lambda_{j},-1}\left[\prod_{j \in \mathcal{L}_{i}}\left(\psi_{c}^{j \rightarrow i} e^{x \gamma}+\psi_{s}^{j \rightarrow i}\right)+\left(e^{x \gamma}-1\right) \prod_{j \in \mathcal{L}_{i}} \psi_{s}^{j \rightarrow i}\right] e^{-x u^{2} / 2} \\
& +\delta_{\Lambda_{j}, A}\left[\prod_{j \in \mathcal{L}_{i}}\left(\psi_{c}^{j \rightarrow i} e^{x \gamma}+\psi_{s}^{j \rightarrow i}\right)\right] .
\end{aligned}
$$

With $\Delta E_{\text {link }}$ from Table 6 , we write $\Xi_{\text {link }}$ as

$$
\Xi_{\text {link }}=\psi_{s}^{L \rightarrow R} \psi_{s}^{R \rightarrow L}+\left(\psi_{s}^{L \rightarrow R} \psi_{c}^{R \rightarrow L}+\psi_{c}^{L \rightarrow R} \psi_{s}^{R \rightarrow L}\right) e^{x \gamma}+\psi_{c}^{L \rightarrow R} \psi_{c}^{R \rightarrow L} e^{x \gamma} .
$$

The configurational free energy $\Phi$ is given by Eq. (68) with $\psi_{s}^{j \rightarrow i}$ averaged over $Q\left(\psi_{c}\right)$. The complexity $\Sigma(\mathrm{e})$ obtained is shown in Fig. 13, Again, e is expressed in terms of the fraction of source nodes through the relation $f_{s}=\left[\mathrm{e}-1 / 2 K+\left(1-\phi_{d}\right) u^{2} / 2\right] / \gamma$.

Generally, we identify three segments on the complexity curve: (i) the unphysical segment (the dotted segment with $\Sigma \geq 0$ ), (ii) the physical segment (the solid segment with $\Sigma \geq 0$ ), and (iii) the highly unlikely segment (the dotted segment with $\Sigma<0$ ). The physical segment of $\Sigma$ is related to the number $\mathcal{N}$ of metastable states with energy e. We denote the values of $x$ when $\Sigma=0$ and $\Sigma$ is maximum as $x_{s}$ and $x_{d}$ respectively. 


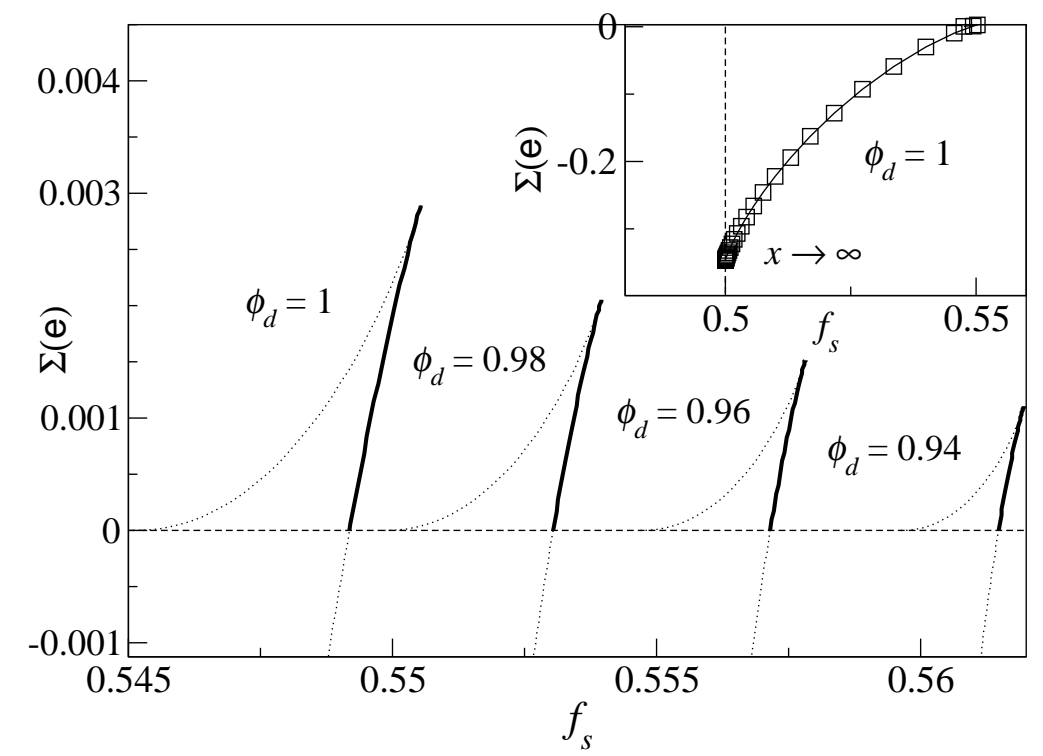

Figure 13. The 1RSB complexity $\Sigma(\mathrm{e})$ obtained by numerically solving for $Q\left(\psi_{c}\right)$ with $K=3$ and $\phi_{d}=0.94,0.96,0.98,1$. Inset: $\Sigma(\mathrm{e})$ for $K=3$ and $\phi_{d}=1$ obtained from the ansatz $Q\left(\psi_{c}\right)=\delta\left(\psi_{c}-\left\langle\psi_{c}\right\rangle\right)$. It approaches the modulation limit at $f_{s}=0.5$ as $x \rightarrow \infty$. Symbols are spaced at intervals of 0.9 in $x \gamma$.

$\mathrm{e}\left(x_{s}\right)$ corresponds to the lowest energy among the states with non-vanishing complexity, which is considered as the ground state in the picture of 1RSB. $\mathrm{e}\left(x_{d}\right)$ corresponds to the energy of the states with the highest complexity, which is believed to be the states where search algorithms get trapped, giving rise to dynamical transitions. However, recent work on the coloring problem shows that the efficacy of the BP algorithm is not affected by the dynamical transition [30]. We leave this issue for future studies.

The segment of negative complexity corresponds to states with vanishing number in the thermodynamic limit. Its physical meaning is clear in the limit $x \rightarrow \infty$, which corresponds to a single state with lowest possible energy, since the reweighting process allows only one state. We show $\Sigma$ in the inset of Fig. 13 as $x \rightarrow \infty$. The result is obtained from the solution of $Q\left(\psi_{c}\right)$ restricted to be the the 1RSB solution in the form of $Q\left(\psi_{c}\right)=\delta\left(\psi_{c}-\left\langle\psi_{c}\right\rangle\right)$, i.e. the unstable solution when $x>x^{(\mathrm{I})}$. The complexity curve approaches the limit of $\psi_{c}=0.5$, which corresponds to the highly unlikely structure of networks with alternating layers of consumer and source nodes.

\subsection{Comparison with Real Instances}

Though successful in finding low lying states on real instances, the GSAT algorithm we described in Section 2.2 requires long computation time for large systems. To compare the predictions of RS and 1RSB approximations with real instances, we employ the $\mathrm{BP}$ with decimation. As the $\mathrm{BP}$ does not converge in the RSB phase, we measure the time average of the fluctuating messages $\psi_{c}^{j \rightarrow i}$ and evaluate for each node the time average probability of the full $\mathcal{S}$-states. The node with the highest $\psi_{\mathcal{S}}^{i}$ is fixed to be 


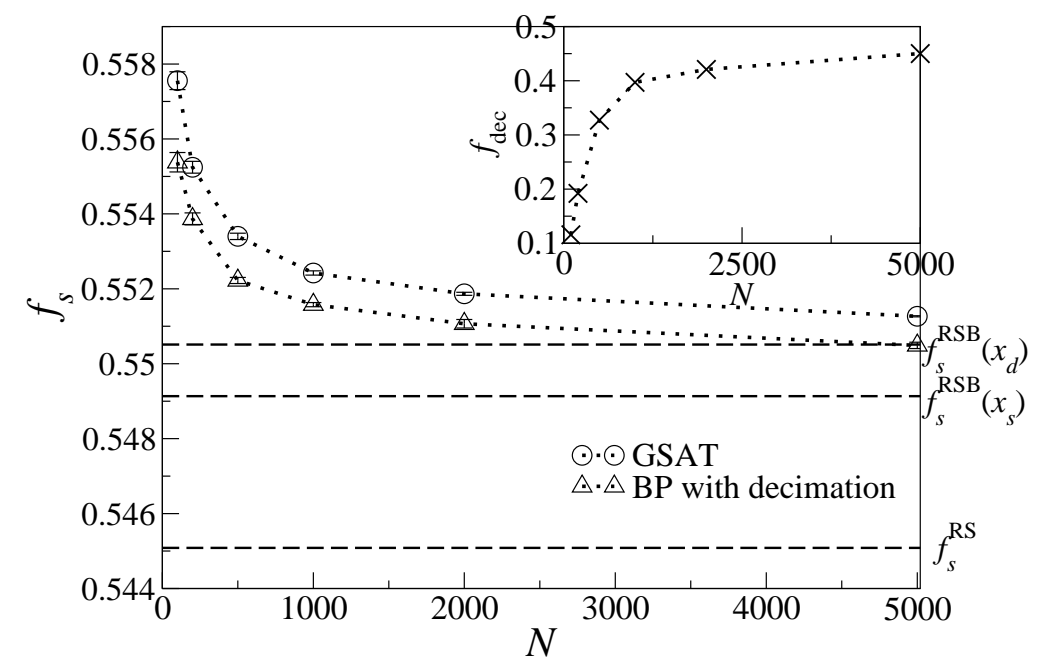

Figure 14. The optimized $f_{s}$ on real instances as a function of $N$ with $K=3$, as obtained by the GSAT algorithm (o) and the BP algorithm with decimation $(\triangle)$. The horizontal dashed lines show the analytical results of the RS ansatz, $f_{s}\left(x_{s}\right)$ and $f_{s}\left(x_{d}\right)$ in the 1RSB ansatz. Inset: the fraction $f_{\text {dec }}$ of decimated nodes before convergence.

resource providing. Only source nodes are decimated, as bistable nodes should be left undecimated, and decimating a consumer node will fix its neighbors simultaneously, which may hinder the convergence of the BP. By repeating the above procedure, the BP messages finally converge and the full state of all nodes are determined.

Figure 14 shows that lower simulated energy can be obtained from the BP with decimation as compared with the GSAT algorithm. The simulated energy approaches the $\mathrm{e}\left(x_{d}\right)$ as obtained by the 1RSB ansatz. However, the fraction of decimated nodes before convergence increases with system size as shown in the inset of Fig. 14. This greatly increases the computational time for large systems if only a single node is decimated at a time. Several nodes can be decimated simultaneously to shorten the computation time, with a tradeoff in energy.

\subsection{The instability of $1 R S B$ formalism}

To test the stability of the 1RSB ansatz against further steps of RSB, we consider two kinds of instability that leads to the two-step RSB (2RSB) formalism [35. They are the so-called type I and type II instabilities, corresponding to the aggregation of states and fragmentation of states respectively, as shown schematically in Fig. 15. In type I instability, metastable states aggregate in clusters and the 1RSB ansatz is valid in each cluster. The whole state space is composed of clusters and the 2RSB formalism is required to describe the state space structure. In type II instability, some states split to form clusters of states instead of single states. The 1RSB ansatz is valid inside the clusters while 2RSB ansatz is required to describe the state space structure. It is generally believed that once 1RSB is not stable, the full RSB is required to describe the system, as illustrated by the 1RSB instability found in graph coloring [36] and $K$ - 


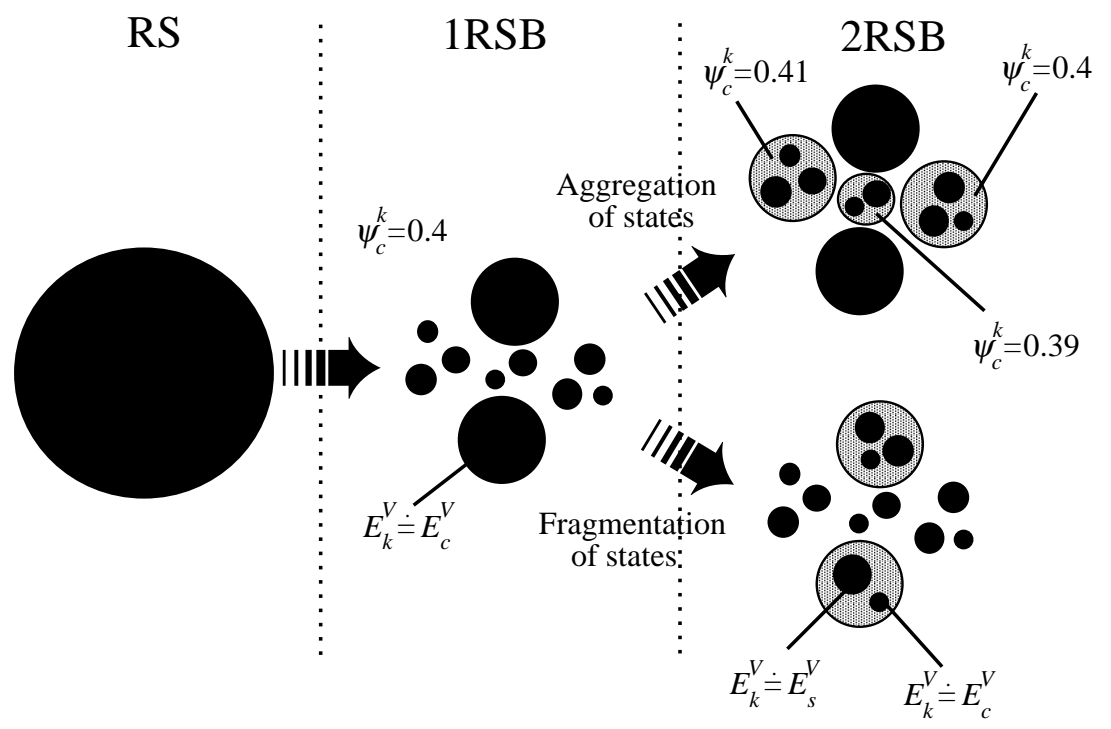

Figure 15. The schematic picture of the state space in the RS, 1RSB and 2RSB phase. Black circles represent singly connected states. Grey circles represent clusters of states. $k$ is an arbitrary vertex in the network. Its probabilities $\psi_{c}^{k}$ of the $C$ states in the 1RSB phase, and several clusters in the 2RSB phase, are shown in the figure to illustrate the aggregation of states. Its cavity energy $E_{k}^{V}$ in an arbitrary state in the 1RSB phase, and several of its fragmented states in the 1RSB phases, are also shown in the figure to illustrate the fragmentation of states. The values of $\psi_{c}^{k}$ are assigned for the purpose of illustration only.

satisfiability problems [37]. Here we focus on the case of $K=3$ in the 1RSB regime with $\phi_{d}>\phi_{d}^{\mathrm{AT}}$, following the approach of [36].

6.4.1. Type I instability: Aggregation of states In the 1RSB phase, each vertex $k$ is characterized by the cavity probability functional $P_{k}\left[E^{V}\right]$. This characterization is much simplified due to the small closed set of states in the singlet regime, rendering it isomorphic to single values of $\psi_{c}^{k}$. In the 2RSB formalism, each vertex $k$ is characterized by the probability functional of $P_{k}$. For the closed set of states in the singlet regime, this characterization is isomorphic to the distribution $Q_{k}\left(\psi_{c}^{k}\right)$. In the example illustrated in Fig. 15, the probability $\psi_{c}^{k}=0.4$ is originally uniform in the state space of the 1RSB phase. When it enters the $2 \mathrm{RSB}$ phase, $\psi_{c}^{k}$ starts to take up diversified values of $\psi_{c}^{k}=0.39,0.40,0.41$ in three different clusters of states.

To examine type I instability, we test for the possible spreading in $\psi_{c}^{j \rightarrow i}$ by introducing small fluctuations in $\psi_{c}^{k \rightarrow j}$ of the descendents. We define $T_{k \rightarrow j}(x)$ to be the derivative

$$
T_{k \rightarrow j}(x)=\left|\frac{\partial \psi_{c}^{j \rightarrow i}}{\partial \psi_{c}^{k \rightarrow j}}\right|_{1 \mathrm{RSB}}
$$

from Eq. (73), which is evaluated at the values of $\psi_{c}^{k \rightarrow j}$ from the 1RSB solution. The 
propagation of noise is thus given by

$$
\left(\delta \psi_{c}^{j \rightarrow i}\right)^{2}=\sum_{k \in \mathcal{L}_{j} \backslash\{i\}}\left[T_{k \rightarrow j}(x)\right]^{2}\left(\delta \psi_{c}^{k \rightarrow j}\right)^{2} .
$$

In the thermodynamic limit, we consider a tree structure with $L$ generations. The noise at the $L^{\text {th }}$ generation vanishes if $\left(T_{L-1 \rightarrow L} T_{L-2 \rightarrow L-1} \ldots T_{0 \rightarrow 1}\right)^{2} \rightarrow 0$ as $L \rightarrow \infty$. Hence we measure the quantity

$$
\lambda_{L}^{(\mathrm{I})}(x)=(K-1)^{L}\left(T_{L-1 \rightarrow L} T_{L-2 \rightarrow L-1} \ldots T_{0 \rightarrow 1}\right)^{2},
$$

averaged over the quenched disorder and boundary conditions of the trees. This can be implemented by iterating Eq. (73) using population dynamics and evaluating the corresponding $\lambda_{L}^{(\mathrm{I})}(x)$ in each iteration. Alternatively, we solve for the distribution $\mathcal{P}_{L}\left[\lambda_{L}^{(\mathrm{I})}, \psi_{c}^{L}\right]$ at layer $L$ by the recursion relation

$$
\begin{aligned}
& \mathcal{P}_{L+1}\left[\lambda_{L+1}^{(\mathrm{I})}, \psi_{c}^{L+1}\right]=\int d \lambda_{L}^{(\mathrm{I})} \int d \psi_{c}^{L} \mathcal{P}_{L}\left[\lambda_{L}^{(\mathrm{I})}, \psi_{c}^{L}\right] \prod_{k=2}^{K-1} \int d \psi_{c}^{k} Q\left(\psi_{c}^{k}\right) \\
& \left\{\phi_{d} \delta\left[\lambda_{L+1}^{(\mathrm{I})}-\left(T_{L \rightarrow L+1}\right)^{2} \lambda_{L}^{(\mathrm{I})}\right] \delta\left[\psi_{c}^{L+1}-\frac{e^{-x u^{2} / 2}}{\mathcal{Z}^{j \rightarrow i}}\left(1-\psi_{c}^{L}\right) \prod_{k=2}^{K-1}\left(1-\psi_{c}^{k}\right)\right]\right. \\
& \left.+\phi_{s} \delta\left(\lambda_{L+1}^{(\mathrm{I})}\right) \delta\left(\psi_{c}^{L+1}\right)\right\}
\end{aligned}
$$

with the initial condition

$$
P_{1}\left[\lambda_{1}^{(\mathrm{I})}, \psi_{c}^{1}\right] \equiv Q\left(\psi_{c}^{1}\right) \delta\left(\lambda_{1}^{(\mathrm{I})}-1\right)
$$

for $L=1$. We note that the case of $\phi_{d}=1$ is a special case where no disorder is present and $T_{0 \rightarrow 1}=\ldots=T_{L-1 \rightarrow L}$ since $Q\left(\psi_{c}^{k}\right)=\delta\left(\psi_{c}^{k}-\left\langle\psi_{c}^{k}\right\rangle\right)$ for all $k$. Thus for $\phi_{d}=1,\left\langle\lambda_{1}^{(I)}(x)\right\rangle \geq 1$ is sufficient to show that 1 RSB solution is unstable at $x$. To evaluate $\left\langle\lambda_{L}^{(\mathrm{I})}(x)\right\rangle$ for general values of $\phi_{d}<1$, solving Eq. (65) gives more reliable results than population dynamics. This is because for large $L$ and nonvanishing values of $\phi_{s}$, such as those close to $\phi_{s}^{\mathrm{AT}}$, the presence of the factor $\prod_{l=1}^{L} \delta_{\Lambda_{l}, A}$ in $\lambda_{L}^{(\mathrm{I})}(x)$ requires an extremely large population for a finite fraction of nonzero $\lambda_{L}^{(\mathrm{I})}(x)$ in the pool of population dynamics.

We show $\left\langle\lambda_{L}^{(\mathrm{I})}(x)\right\rangle$ as a function of $L$ in Fig. 16(a) for $\phi_{d}=0.9$ from $x \gamma=0$ to $x \gamma=4.5$, and define $x^{(\mathrm{I})}$ by

$$
\left.\lim _{L \rightarrow \infty} \frac{d \log \left\langle\lambda_{L}^{(\mathrm{I})}(x)\right\rangle}{d L}\right|_{x=x^{(\mathrm{I})}}=1 .
$$

From Fig. 16(a), the 1RSB solution for $\phi_{d}=0.9$ is stable against type I instability when $x \gamma<x^{(\mathrm{I})} \gamma \approx 3.07$

6.4.2. Type II instability: Fragmentation of states In the 1 RSB phase, vertex $k$ is characterized by a cavity energy function $E_{k}^{V}$ in each single state. In the 2RSB phase, some 1RSB single states split into different states in which some vertices are characterized by more than one cavity energy functions. In the example illustrated in 
Fig. 15, a state with $E_{k}^{V}=E_{c}^{V}$ is fragmented to states with $E_{k}^{V}=E_{c}^{V}$ and $E_{k}^{V}=E_{s}^{V}$ respectively on entering the $2 \mathrm{RSB}$ phase.

As the cavity energy functions of some vertices are modified during fragmentation, we examine the probability of changes in $E_{i}^{V}$ of a node $i$ due to changes in $E_{j}^{V}$ among its descendent nodes $j$, the so-called proliferation of bugs [36]. In the singlet regime, we denote $\pi_{s \rightarrow c}^{k \rightarrow j}$ as the joint probability that vertex $k \rightarrow j$ is in the $S$ state in the absence of bugs, and in the $C$ state in the presence of a small number of bugs. From the recursion Eq. (73), we note that contributions to $\pi_{c \rightarrow s}^{j \rightarrow i}$ come from the case that all descendents of $j$ are in the $S$ state, and one of them changes to $C$ state in the presence of bugs. Hence

$$
\pi_{c \rightarrow s}^{j \rightarrow i}=\delta_{\Lambda_{j},-1} \frac{e^{-x u^{2} / 2}}{\mathcal{Z}^{j \rightarrow i}} \sum_{k \in \mathcal{L}_{j} \backslash\{i\}} \pi_{s \rightarrow c}^{k \rightarrow j} \prod_{l \in \mathcal{L}_{j} \backslash\{i, k\}} \psi_{s}^{l \rightarrow j} e^{x \gamma} .
$$

Similarly, contributions to $\pi_{s \rightarrow c}^{j \rightarrow i}$ come from the case that only one descendent of $j$ is in the $C$ state which changes to $S$ state in the presence of bugs. Hence

$$
\pi_{s \rightarrow c}^{j \rightarrow i}=\delta_{\Lambda_{j},-1} \frac{e^{-x u^{2} / 2}}{\mathcal{Z}^{j \rightarrow i}} \sum_{k \in \mathcal{L}_{j} \backslash\{i\}} \pi_{c \rightarrow s}^{k \rightarrow j} \prod_{l \in \mathcal{L}_{j} \backslash\{i, k\}} \psi_{s}^{l \rightarrow j} .
$$

We define the matrix $V_{k \rightarrow j}(x)$ to be

$$
V_{k \rightarrow j}(x)=\delta_{\Lambda_{j},-1} \frac{e^{-x u^{2} / 2}}{\mathcal{Z}^{j \rightarrow i}}\left(\begin{array}{ccc}
0 & \prod_{l \in \mathcal{L}_{j} \backslash\{i, k\}} \psi_{s}^{l \rightarrow j} & \psi_{s}^{l \rightarrow j} e^{x \gamma} \\
\prod_{l \in \mathcal{L}_{j} \backslash\{i, k\}} & 0
\end{array}\right)
$$

The instability of the 1RSB solution against fragmentation of states can be thus examined by considering the maximum eigenvalue of the products of matrices $V$. We measure the quantity

$$
\lambda_{L}^{(\mathrm{II})}(x)=(K-1)^{L} \mathcal{I}\left(V_{L-1 \rightarrow L} V_{L-2 \rightarrow L-1} \ldots V_{0 \rightarrow 1}\right),
$$

averaged over quenched disorders, where $\mathcal{I}(\ldots)$ is defined as the maximum eigenvalue of the matrix. The 1 RSB solution is stable against the type II instability if $\left\langle\lambda_{L}^{(\text {II })}(x)\right\rangle \rightarrow 0$ as $L \rightarrow \infty$. $\left\langle\lambda_{L}^{(\mathrm{II})}(x)\right\rangle$ can be solved by population dynamics of Eq. (73) or by solving for the distribution on $\mathcal{P}_{L}\left[\lambda_{L}^{(\mathrm{II})}, \psi_{c}^{L}\right]$ analogous to Eq. (79)).

We show $\left\langle\lambda_{L}^{(\mathrm{II})}(x)\right\rangle$ as a function of $L$ in Fig. 16(b) for $\phi_{d}=0.9$ from $x \gamma=0$ to $x \gamma=4.5$, and define $x^{(\mathrm{II})}$ similarly as Eq. (81). From Fig. 16)(b), the 1RSB solution for $\phi_{d}=0.9$ is stable against type II instability when $x \gamma<x^{(\mathrm{II})} \gamma \approx 2.93$.

$x^{(\mathrm{I})}$ and $x^{(\mathrm{II})}$ from $\phi_{d}=0.78$ to 1 , together with $x_{s}$ and $x_{d}$ obtained from the complexity curve, are plotted in Fig. 16(c) as a function of $\phi_{d}$. Reliable results for $\phi_{d} \approx \phi_{d}^{\mathrm{AT}}=0.75$ are difficult to obtain as the continuous component in $Q\left(\psi_{c}\right)$ becomes extremely small (see for instance, Fig. 12) and extremely high precision is required. For $\phi_{d}$ lower than $\approx 0.88$, the 1 RSB ansatz is stable in the range of $x^{(\mathrm{II})}<x<x^{(\mathrm{I})}$, corresponding to the shaded region in Fig. 16(c). Both $x_{s}$ and $x_{d}$ are found below the shaded region, indicating the instability of the physical segment of $\Sigma$. The stable range of $x$ lies on the negative segment of $\Sigma$, which implies that states with vanishing 

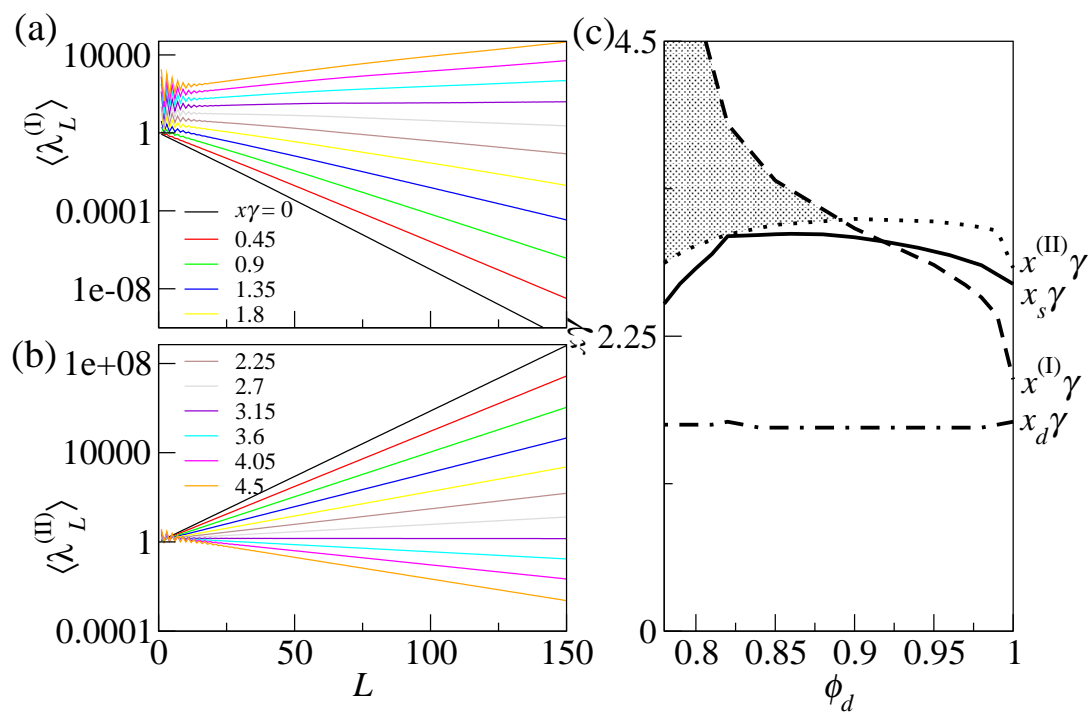

Figure 16. (a) $\left\langle\lambda_{L}^{(\mathrm{I})}(x)\right\rangle$ and (b) $\left\langle\lambda_{L}^{(\mathrm{II})}(x)\right\rangle$ as a function of $L$ for systems with $K=3$ and $\phi_{d}=0.9$, from $x \gamma=0$ to $x \gamma=4.5$. (c) $x^{(\mathrm{I})} \gamma, x^{(\mathrm{II})} \gamma, x_{s} \gamma$ and $x_{d} \gamma$ as a function of $\phi_{d}$ for $K=3$. The shaded region corresponds to the stable range of the 1RSB ansatz.

probability are stable in the $1 \mathrm{RSB}$ ansatz. For $\phi_{d}$ higher than $\approx 0.88$, the shaded region disappears and all complexity curves are unstable. We thus conclude that the 1RSB quantities evaluated at $x_{s}$ and $x_{d}$ are unstable. Nevertheless, agreement with simulation results show that the 1RSB ansatz is a good approximation of the physical picture of the system.

\section{Conclusion}

In this paper, we have studied the source location problem on transportation networks. As the formulation involves continuous variables, the cavity fields are represented by the cavity energy functions which satisfy the piecewise quadratic ansatz. The ansatz decomposes the cavity energy functions into composite functions, and effectively parametrizes them by the energy minimum of each composite function.

This enables us to obtain a small closed set of cavity energy functions in the singlet regime, which greatly simplifies the functional RS recursions to simple recursions of probabilities. Physical results such as the average energy and the fraction of soft nodes are obtained and have excellent agreement with simulations when the fraction of deficient nodes is small. We examined the stability of the RS solution and derive the AT-line for the transition to the RSB phase. In the high connectivity limit, such results are consistent with the RS instability obtained in the vertex cover problem for Poissonian graphs.

Though the solution of the 1RSB ansatz corresponds to a stable distribution of functionals, which is in general infeasible to solve, the closed sets of cavity energy functions greatly simplify the 1RSB recursions which make the 1RSB solution feasible. We remark that the analysis is applicable to regimes other than the singlet regime of 
the system, such as the commensurate points of the doublet regime being considered in Appendix A. In other cases, closed sets with a large number of functions are found and the 1RSB solution may once again become computational infeasible. We expect that the present techniques are applicable to other problems where closed sets of cavity fields exist.

\section{Appendix A. The doublet Regime}

Appendix A.1. The Closed Set of Cavity Energy Functions and the Simplified RS Recursion

Apart from the closed set of cavity energy functions $E^{V}$ in the singlet regime, a closed set with countable elements of $E^{V}$ can also be found in other regimes. In the doublet regime, we find that a small number of $E^{V}$ spans a closed set at the commensurate points

$$
\gamma=m \kappa,
$$

with the rational number $m \geq 2$. These commensurate points correspond to the values of

$$
u^{-1}=-\sqrt{\frac{K(K-1)(m-1)}{(m-1) K+m+1}},
$$

at which abrupt jumps in the fraction of source nodes are found in the range $\sqrt{21 / 25}<$ $u^{-1}<\sqrt{3 / 2}$ in Fig. 2 for $K=3$. Each value of rational $m$ corresponds to a switch of energetic stability from one configuration of consumer nodes to another.

To find the closed set of $E^{V}$ in the doublet regime, we only have to consider the composite functions $f_{0}, f_{1}$ and $f_{2}$. $E_{k}^{V}\left(y_{k}\right)$ is thus denoted as

$$
E_{k}^{V}\left(y_{k}\right) \doteq\left(d_{0}^{k}, d_{1}^{k}, d_{2}^{k}\right) .
$$

All other composite functions $f_{n_{k}}$ with $n_{k} \geq 3$ have $d_{n_{k}}^{k}>d_{n_{k}^{*}}^{k}$ and their corresponding configurations are not stable in the doublet regime. We thus consider only the recursion $\mathcal{M}(0, \ldots, 0)=1$ and $\mathcal{M}(1,0, \ldots, 0)=2$. As an illustration, we consider the case $m=2$ and $\phi_{d}=1$ where closed set of $E^{V}$ is shown in Fig. A1. The cavity energy change $\Delta E_{j}$ from Eq. (30), the constant terms $d_{0}^{j}, d_{1}^{j}$ and $d_{2}^{j}$ from Eqs. (22) and (29) are simplified to

$$
\begin{aligned}
\Delta E_{j} & =\frac{u^{2}}{2}+\sum_{k \in \mathcal{L}_{j} \backslash\{i\}} \min \left(0, d_{1}^{k}, d_{2}^{k}\right), \\
d_{0}^{j} & =0, \\
d_{1}^{j} & =-2 \kappa-\sum_{k \in \mathcal{L}_{j} \backslash\{i\}} \min \left(0, d_{1}^{k}, d_{2}^{k}\right), \\
d_{2}^{j} & =-\kappa+\min _{k \in \mathcal{L}_{j} \backslash\{i\}} d_{1}^{k}-\sum_{k \in \mathcal{L}_{j} \backslash\{i\}} \min \left(0, d_{1}^{k}, d_{2}^{k}\right),
\end{aligned}
$$



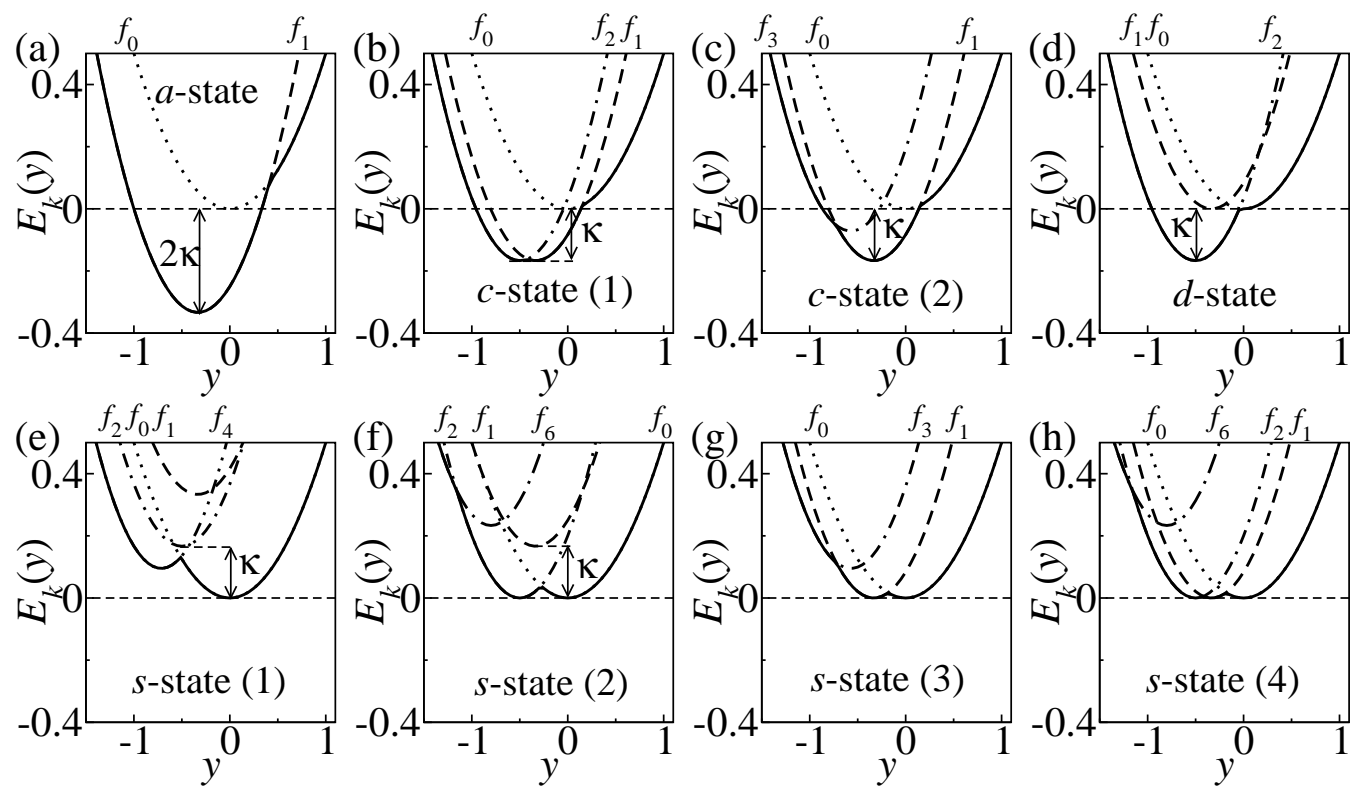

Figure A1. A closed set of cavity energy functions $E_{k}(y)$ at $K=3, u^{-1}=1$ in the doublet regime. The forms of $E^{V}$ corresponds to (a) the $a$ state, (b-c) the $c$ state, (d) the $d$ state and (e-h) the $s$ state.

where the zero-point of $E^{V}$ is made at the minimum values of $f_{0}$ for convenience of analysis. The energy change of $\Delta E_{j}$ of adding vertex $j$ is obtained by comparing the energies of the $S$ states of the vertex and its descendents, and can be shown to produce the same physical results as the full energetic comparison. From the recursion relations, the full closed set of $E^{V}$ is found to be

$$
\begin{aligned}
& E_{a}^{V}(y) \doteq(0,-2 \kappa,(-1+r) \kappa) \\
& E_{c}^{V}(y) \doteq(0,-\kappa,(-1+r) \kappa) \\
& E_{d}^{V}(y) \doteq(0, r \kappa,-1 \kappa) \\
& E_{s}^{V}(y) \doteq(0, q \kappa, r \kappa)
\end{aligned}
$$

with integers $q, r \geq 0$. $E_{a}^{V}$ corresponds to cavity states with a strong preference to be singly consuming. $E_{c}^{V}, E_{d}^{V}$ and $E_{s}^{V}$ correspond to cavity states which are respectively singly consuming, doubly consuming and resource providing. They are denoted as the $a, c, d$ and $s$ states. Note that the integer $r \geq 1$ in Eq. (A.5) may correspond to the form of $E^{V}$ where $f_{1}$ and $f_{2}$ are not relevant (see for instance Fig. A1 (f) and (g)). For $K=3$, there are two forms of $E_{c}^{V}$ with $r=0$ and $r \geq 1$ and four forms of $E_{s}^{V}$ with $(q, r)$ $=(\geq 1,1),(\geq 1,0),(0, \geq 1)$ and $(0,0)$. Hence the closed set of $E^{V}$ has eight forms of $E^{V}$ as shown in Fig. A1,

Next, we consider the disordered case $\phi_{d}<1$. The recursion relations can be simplified in terms of $\psi_{a}, \psi_{c}, \psi_{d}$ and $\psi_{s}$, corresponding to the probabilities for a vertex to be in the $a, c, d$ and $s$ states, as given by

$$
\psi_{a}^{j \rightarrow i}=\delta_{\Lambda_{j},-1} \prod_{k \in \mathcal{L}_{j} \backslash\{i\}} \psi_{s}^{k \rightarrow j},
$$



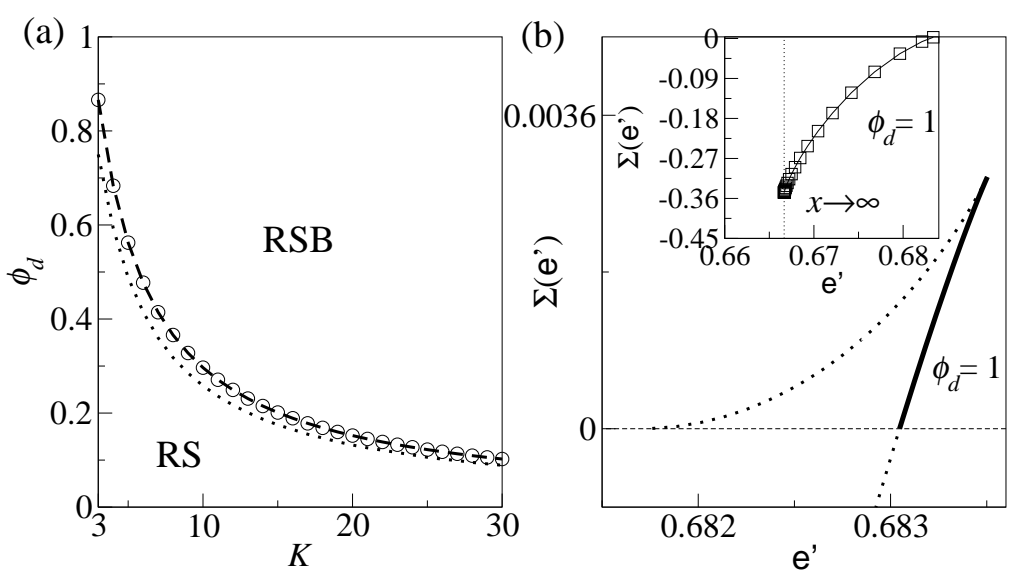

Figure A2. (a) The $K-\phi_{d}$ phase diagram and the AT line (dashed line with circles) in the doublet regime with $u^{-1}=\sqrt{K(K-1) /(K+3)}$. The dotted line is the AT line in the singlet regime. (b) The complexity function $\Sigma(\mathrm{e})$ from the 1RSB solution for $K=3$ with $u^{-1}=1$. $\mathrm{e}^{\prime}=\mathrm{e} /\left(u^{2} / 2\right)$. Inset: The negative segment of the complexity function shown in the limit $x \rightarrow \infty$. Each symbol on the line indicates an increase of 1.2 in $x \kappa$.

$$
\begin{aligned}
& \psi_{c}^{j \rightarrow i}=\delta_{\Lambda_{j},-1} \sum_{k \in \mathcal{L}_{j} \backslash\{i\}}\left(\psi_{c}^{k \rightarrow j}+\psi_{d}^{k \rightarrow j}\right) \prod_{l \in \mathcal{L}_{j} \backslash\{i, k\}} \psi_{s}^{l \rightarrow j}, \\
& \psi_{d}^{j \rightarrow i}=\delta_{\Lambda_{j},-1} \sum_{k \in \mathcal{L}_{j} \backslash\{i\}} \psi_{a}^{k \rightarrow j} \prod_{l \in \mathcal{L}_{j} \backslash\{i, k\}} \psi_{s}^{l \rightarrow j},
\end{aligned}
$$

and $\psi_{s}^{j \rightarrow i}=1-\psi_{a}^{j \rightarrow i}-\psi_{c}^{j \rightarrow i}-\psi_{d}^{j \rightarrow i}$ In the RS phase, we set $\psi_{a}^{j \rightarrow i}, \psi_{c}^{j \rightarrow i}, \psi_{d}^{j \rightarrow i}, \psi_{s}^{j \rightarrow i}=0,1$. The average optimized energy in the RS ansatz is obtained by evaluating the full energy change $\Delta \mathcal{E}_{\text {node }}$ and $\Delta \mathcal{E}_{\text {link }}$ resulting from the addition of new nodes and new links. The expression for $\Delta \mathcal{E}_{\text {node }}$ and $\Delta \mathcal{E}_{\text {link }}$ from Eq. (8) can be simplified as

$$
\begin{aligned}
& \Delta \mathcal{E}_{\text {node }}^{i}=\delta_{\Lambda_{i},-1}\left\{\frac{u^{2}}{2}+\min \left[\sum_{j \in \mathcal{L}_{i}} \min \left(0, d_{1}^{j}, d_{2}^{j}\right),-2 \kappa,-\kappa+\min _{j \in \mathcal{L}_{i}} d_{1}^{j}\right]\right\} \\
& +\delta_{\Lambda_{i}, A} \sum_{j \in \mathcal{L}_{i}} \min \left(0, d_{1}^{j}, d_{2}^{j}\right) . \\
& \Delta \mathcal{E}_{\text {link }}=\min \left[0, d_{1}^{L}, d_{1}^{R}, \frac{1}{K(K-1)}+d_{1}^{L}+d_{1}^{R}, d_{2}^{L}, d_{2}^{R}\right] .
\end{aligned}
$$

\section{Appendix A.2. The Instability of the RS Ansatz}

To obtain the AT line between the RS and the RSB phases, we consider the variations $\delta \psi_{a}^{k \rightarrow j}, \delta \psi_{c}^{k \rightarrow j}, \delta \psi_{d}^{k \rightarrow j}$ and $\delta \psi_{s}^{k \rightarrow j}$ in the recursions of probabilities in Eq. (A.6), with $\delta \psi_{a}^{k \rightarrow j}+\delta \psi_{c}^{k \rightarrow j}+\delta \psi_{d}^{k \rightarrow j}+\delta \psi_{s}^{k \rightarrow j}=0$. Though the $c$ and $d$ states lead to different degeneracies of the full states, the two states play the same role in the recursion relations and lead to the same $\Delta E, \Delta \mathcal{E}_{\text {node }}$ and $\Delta \mathcal{E}_{\text {link. }}$. Combining $\psi_{c}^{k \rightarrow j}$ and $\psi_{d}^{k \rightarrow j}$ in the recursions, the variations $\delta \psi_{a}^{k \rightarrow j}, \delta\left(\psi_{c}^{k \rightarrow j}+\psi_{d}^{k \rightarrow j}\right)$ and $\delta \psi_{s}^{k \rightarrow j}$ depends only on $\delta \psi_{s}^{k \rightarrow j}$ of the descendents. We thus write down the AT condition for the disordered case 
of the doublet regime,

$$
\frac{\left\langle\left(\delta \psi_{s}^{j \rightarrow i}\right)^{2}\right\rangle}{\left\langle\left(\delta \psi_{s}^{k \rightarrow j}\right)^{2}\right\rangle}=(K-1)(K-2) \phi_{d}\left(1-\left\langle\psi_{s}^{k \rightarrow j}\right\rangle\right)\left\langle\psi_{s}^{k \rightarrow j}\right\rangle^{K-3},
$$

where we have applied the relation of $\left\langle\left(\psi_{s}^{k \rightarrow j}\right)^{2}\right\rangle=\left\langle\psi_{r}^{k \rightarrow j}\right\rangle$ in the derivation. Simple algebra leads to the following form of the AT line

$$
\begin{aligned}
& \phi_{d}^{\mathrm{AT}}=\frac{1}{(K-1)(K-2)\left(1-\left\langle\psi_{s}\right\rangle^{\mathrm{AT}}\right)\left(\left\langle\psi_{s}\right\rangle^{\mathrm{AT}}\right)^{K-3}}, \\
& \left\langle\psi_{s}\right\rangle^{\mathrm{AT}}=\frac{(2 K-3)(K-1)-\sqrt{(K-1)(5 K-9)}}{2 K(K-2)},
\end{aligned}
$$

where $\left\langle\psi_{s}\right\rangle^{\mathrm{AT}}$ is the average value of $\psi_{s}$ on the AT line. The $K-\phi_{d}$ phase diagram is shown in Fig. A2(a), which is compared to the phase diagram of the singlet regime in Fig. 11 inset. The comparison suggests that the RSB phase shrinks when $u^{-1}$ decreases from the singlet regime to the doublet regime.

\section{Appendix A.3. The 1RSB solution}

To obtain the 1RSB solution, we follow the approach adopted in the singlet regime and evaluate the partition functions $\Xi_{\text {node }}$ and $\Xi_{\text {link }}$ using the full energy change $\mathcal{E}_{\text {node }}$ and $\mathcal{E}_{\text {link }}$. We solve the 1 RSB solution for the case without disorder (i.e. $\phi_{d}=1$ ) with the $1 \mathrm{RSB}$ restriction on delta functional form of $\mathcal{Q}\left\{P\left[E^{V}\right]\right\}$, i.e. $Q\left(\psi_{a}, \psi_{c}, \psi_{d}\right)=\delta\left(\psi_{a}-\right.$ $\left.\left\langle\psi_{a}\right\rangle\right) \delta\left(\psi_{c}-\left\langle\psi_{c}\right\rangle\right) \delta\left(\psi_{d}-\left\langle\psi_{d}\right\rangle\right)$. The complexity function $\Sigma$ is obtained from the 1RSB solution for $K=3$, and is shown in in Fig. A2(b). Compared with the singlet regime, $\Sigma$ in both cases have similar form and similar maximum values. The physical segment of the curve is shown by the solid segment. Note that $\mathrm{e}=\left(1-f_{\mathcal{C}}-f_{\mathcal{D}}\right) / 2+f_{\mathcal{C}} / 6+f_{\mathcal{D}} / 3$ and the fraction $f_{\mathcal{C}}$ and $f_{\mathcal{D}}$ of singly and doubly consuming nodes are not uniquely determined by the energy e. The negative segment of the complexity function approaches the limit of $\mathrm{e}=1 / 3$ as $x \rightarrow \infty$, as shown in the inset of Fig. A2(b). It corresponds to the lowest possible energy on graphs with special structures, which occur with vanishing probability in the thermodynamic limit. $\mathrm{e}=1 / 3$ implies $4 f_{\mathcal{C}}+3 f_{\mathcal{D}}=2$, suggesting the modulation limit of $f_{\mathcal{C}}=1 / 2$ when $f_{\mathcal{D}}=0$ as in the singlet regime, or the limit of $f_{\mathcal{D}}=2 / 3$ when $f_{\mathcal{C}}=0$ where two-third of nodes are doubly consuming.

\section{Acknowledgements}

This work is supported by the Research Grants Council of Hong Kong (grant numbers HKUST 603607 and HKUST 604008).

[1] M. R. Garey and D. S. Johnson, Computers and Intractability: A Guide to the Theory of NPCompleteness (Freeman, New York, 1979).

[2] M. Mézard, G. Parisi and M. A. Virasoro, Spin Glass Theory and Beyond (World Scientific, 1987).

[3] H. Nishimori, Statistical Physics of Spin Glasses and Information Processing (Oxford University Press, Oxford, UK, 2001).

[4] M. Mézard and R. Zecchina, Phys. Rev. E 66, 056126 (2002). 
[5] R. Mulet, A. Pagnani, M. Weigt, and R. Zecchina, Phys. Rev. Lett. 89, 268701 (2002); A. Braunstein, R. Mulet, A. Pagnani, M. Weigt, and R. Zecchina, Phys. Rev. E 68, 036702 (2003).

[6] M. Weigt and A. K. Hartmann, Phys. Rev. Lett. 84, 6118 (2000).

[7] M. Weigt and A. K. Hartmann, Phys. Rev. E 63, 056127 (2001).

[8] H. Zhou, Phys. Rev. Lett. 94, 217203 (2005).

[9] K. Y .M. Wong and D. Saad, Phys. Rev. E 74, 010104 (2006).

[10] K. Y .M. Wong and D. Saad, Phys. Rev. E 76, 011115 (2007).

[11] C. H. Yeung and K. Y. M. Wong, J. Stat. Mech, P03029 (2009).

[12] C. Kopparapu, Load Balancing Servers, Firewalls and Caches (Wiley, 2002).

[13] R. L. Rardin Optimization in Operations Research (Prentice Hall, Englewood Cliffs, NJ, 1998).

[14] C. H. Yeung and K. Y. M. Wong, Phys. Rev. E 80, 021102 (2009).

[15] K. Y. M. Wong and D. Sherrington, J. Phys. A 23, L175 (1990).

[16] K. Y. M. Wong and D. Sherrington, Phys. Rev. E 47, 4465 (1993); erratum, Phys. Rev. E 50, $1727(1994)$.

[17] W. Whyte, D Sherrington and K. Y. M. Wong, J. Phys. A 28, 7105 (1995).

[18] P. Luo and K. Y. M. Wong, Phys. Rev. E 64, 061912 (2001).

[19] Y. Imry and S. K. Ma, Phys. Rev. Lett. 50, 1399 (1975).

[20] R. Bruinsma and G. Aeppli, Phys. Rev. Lett. 50, 1494 (1983).

[21] R. Bruinsma, Phys. Rev. B 30, 289 (1984).

[22] G. Biroli and M. Mézard, Phys. Rev. Lett. 88, 025501 (2002).

[23] O. Rivoire, G. Biroli, O. C. Martin, and M. Mézard, Eur. Phys. J. B 37, 55 (2004).

[24] F. Krzakala, M. Tarzia, and L. Zdeborová, Phys. Rev. Lett. 101, 165702 (2008).

[25] G. Toulouse, Comm. on Phys. 2, 115 (1977).

[26] R. L. Devaney, An Introduction to Chaotic Dynamical Systems (Addison-Wesley, Redwood City, CA, 1989).

[27] Y. Weiss and W. T. Freeman, Neural Computation 13, 2173 (2001).

[28] D. Bickson, D. Dolev, O. Shental, P. H. Siegel, and J. K. Wolf, The 2008 International Symposium on Information Theory (ISIT2008), Toronto, 2008.

[29] J. Pearl, Probabilistic Reasoning in Intelligent Systems: Networks of Plausible Inference (Morgan Kaufmann, San Mateo, CA, 1988).

[30] L. Zdeborová and F. Krzakala, Phys. Rev. E 76, 031131 (2007).

[31] B. Selman, H. Kautz and B. Cohen DIMACS Series in Discrete Mathematics and Theoretical Computer Science 26, 521 (1996).

[32] M. Mézard and G. Parisi, Eur. Phys. J. B 20, 217 (2001).

[33] M. Mézard and G. Parisi, J. Stat. Phys. 111, 112 (2003).

[34] D. J. Thouless, Phys. Rev. Lett. 56, 1082 (1986).

[35] A. Montanari and F.Ricci-Tersenghi, Eur. Phys. J. B 33, 339 (2003).

[36] F. Krzakala, A. Pagnani and Martin Weigt, Phys. Rev. E 70, 046705 (2004).

[37] A. Montanari, G. Parisi and F. Ricci-Tersenghi, J. Phys. A: Math. Gen. 37, 2073 (2004). 